$\mathrm{PM}-02-39$

CERN TH/2002-325

November 2002

(updated April 2005)

\title{
SuSpect: a Fortran Code for the Supersymmetric and Higgs Particle Spectrum in the MSSM*
}

\author{
Abdelhak DJOUADI ${ }^{1,2}$, JeAn-LoÏC KNEUR $^{1}$ and Gilbert MOULTAKA ${ }^{1}$ \\ ${ }^{1}$ Laboratoire de Physique Théorique et Astroparticules, UMR5207-CNRS, \\ Université de Montpellier II, F-34095 Montpellier Cedex 5, France. \\ ${ }^{2}$ Laboratoire de Physique Théorique d'Orsay, UMR8627-CNRS, \\ Université Paris-Sud, Bât. 210, F-91405 Orsay Cedex, France.
}

\begin{abstract}
We present the ForTRAN code SuSpect version 2.3, which calculates the Supersymmetric and Higgs particle spectrum in the Minimal Supersymmetric Standard Model (MSSM). The calculation can be performed in constrained models with universal boundary conditions at high scales such as the gravity (mSUGRA), anomaly (AMSB) or gauge (GMSB) mediated supersymmetry breaking models, but also in the nonuniversal MSSM case with R-parity and CP conservation. Care has been taken to treat important features such as the renormalization group evolution of parameters between low and high energy scales, the consistent implementation of radiative electroweak symmetry breaking and the calculation of the physical masses of the Higgs bosons and supersymmetric particles taking into account the dominant radiative corrections. Some checks of important theoretical and experimental features, such as the absence of non desired minima, large fine-tuning in the electroweak symmetry breaking condition, as well as agreement with precision measurements can be performed. The program is simple to use, self-contained and can easily be linked to other codes; it is rather fast and flexible, thus allowing scans of the parameter space with several possible options and choices for model assumptions and approximations.
\end{abstract}

\footnotetext{
${ }^{*}$ The program with all relevant information can be downloaded from the web at the http site: www.lpta.univ-montp2.fr/ ${ }^{\sim}$ nneur/Suspect or obtained by sending an E-mail to one of the authors, abdelhak.djouadi@cern.ch, kneur@lpta.univ-montp2.fr, moultaka@lpta.univ-montp2.fr.
} 


\section{Introduction}

Supersymmetric theories (SUSY) [1], which provide an elegant way to stabilize the large hierarchy between the Grand Unification (GUT) and the electroweak scales and to cancel the quadratic divergences of the radiative corrections to the Higgs boson masses, are by far the most studied extensions of the Standard Model (SM). The most economical low-energy SUSY extension of the SM, the Minimal Supersymmetric Standard Model (MSSM), which allows for a consistent unification of the SM gauge couplings and provides a natural solution of the Dark Matter problem, has been widely investigated; for reviews see Refs. [2-5]. As a corollary, the search for Supersymmetric particles and for the extended Higgs spectrum has become the main goal of present and future high-energy colliders [6].

It is well-known that in the unconstrained MSSM, it is a rather tedious task to deal with the basic parameters of the Lagrangian and to derive in an exhaustive manner their relationship with the physical parameters, i.e. the particle masses and couplings. This is mainly due to the fact that in the MSSM, despite of the minimal gauge group, minimal particle content, minimal couplings imposed by R-parity conservation and the minimal set of soft SUSY-breaking parameters, there are more than a hundred new parameters [7]. Even if one constrains the model to have a viable phenomenology [we will call later such a model the phenomenological MSSM], assuming for instance no intergenerational mixing to avoid flavor changing neutral currents, no new source of $\mathrm{CP}$ violation, universality of first and second generation sfermions to cope with constraints from kaon physics, etc.., there are still more than 20 free parameters left. This large number of input enters in the evaluation of the masses of $\mathcal{O}(30)$ SUSY particles and Higgs bosons as well as their complicated couplings, which involve several non-trivial aspects, such as the mixing between different states, the Majorana nature of some particles, etc. The situation becomes particularly difficult if one aims at rather precise calculations and hence, attempts to include some refinements such as higher order corrections, which for the calculation of a single parameter need the knowledge of a large part of, if not the whole, spectrum.

Thus, the large number of free parameters in the unconstrained or even phenomenological MSSM, makes a detailed phenomenological analysis of the spectra and the comparison with the outcome or expectation from experiment, a daunting task, if possible at all. Fortunately, there are well motivated theoretical models where the soft SUSY-breaking parameters obey a number of universal boundary conditions at the high (GUT) scale, leading to only a handful of basic parameters. This is the case for instance of the minimal Supergravity model (mSUGRA) [8], where it is assumed that SUSY-breaking occurs in a hidden sector which communicates with the visible sector only through "flavor-blind" gravitational interactions. This leads to the simpler situation where the entire spectrum of superparticles and Higgs bosons is determined by the values of only five free parameters and makes comprehensive scans of the parameter space and detailed studies of the spectrum feasible. 
However, there are also similarly constrained and highly predictive alternative SUSYbreaking models in the literature, such as anomaly mediated [9-11] or gauge mediated $[12,13]$ SUSY-breaking models for instance, which should be investigated as well. We then have to trade a complicated situation where we have one model with many input parameters, with a not less complicated situation where we have many models with a small number of basic parameters. In addition, in these unified models, the low-energy parameters are derived from the high-energy (GUT and/or possibly some intermediate scales) input parameters through Renormalization Group Equations (RGE) and they should also necessarily involve radiative electroweak symmetry breaking (EWSB), which sets additional constraints on the model. The implementation of the RG evolution and the EWSB mechanism poses numerous non-trivial technical problems if they have to be done in an accurate way, i.e. including higher order effects. This complication has to be added to the one from the calculation of the particle masses and couplings with radiative corrections (RC) which is still present.

Therefore, to deal with the supersymmetric spectrum in all possible cases, one needs very sophisticated programs to encode all the information and, eventually, to pass it to other programs or Monte Carlo generators to simulate the physical properties of the new particles, decay branching ratios, production cross sections at various colliders, etc... These programs should have a high degree of flexibility in the choice of the model and/or the input parameters and an adequate level of approximation at different stages, for instance in the incorporation of the RGEs, the handling of the EWSB and the inclusion of radiative corrections to (super)particle masses, which in many cases can be very important. They should also be reliable, quite fast to allow for rapid comprehensive scans of the parameter space and simple enough to be linked with other programs. There are several public codes, in particular ISASUGRA [14], SOFTSUSY [15] and SPHENO [16], as well as a number of private codes, which deal with this problem. In this paper we present our program SuSpect.

SuSpect, in its latest version 2.3 that we present here, is a FORTRAN code which calculates the supersymmetric and Higgs particle spectrum in the constrained and unconstrained MSSMs. The acronym is an abbreviation of SusySpectrum and successive previous public versions of the code were available starting from 1997 and have been described in Ref. [17]. At the present stage, it deals with the "phenomenological MSSM" with 22 free parameters defined either at a low or high energy scale, with the possibility of RG evolution to arbitrary scales, and the most studied constrained models, namely mSUGRA, AMSB and GMSB. Many "intermediate" models [e.g. constrained models but without unification of gaugino or scalar masses, etc..] are easily handled. The program includes the three major ingredients which should be incorporated in any algorithm for the constrained MSSMs: $i$ ) renormalization group evolution of parameters between a low energy scale [e.g. $M_{Z}$ and/or the EWSB scale] and a high-energy scale [e.g. $M_{\mathrm{GUT}}$ ], [17-19]; ii) consistent implementation of radiative electroweak symmetry breaking [loop corrections to the effective potential are included using the tadpole method] [20-23]; iii) calculation of the physical (pole) masses 
of the superparticles and Higgs bosons, including all relevant features such as the mixing between various states and the radiative corrections when important [24-34].

The present version includes new options to read input files and write output files in the recently adopted format of the SUSY Les Houches Accord (SLHA) for interfacing the spectrum generators with other computer codes [35]. The code contains three types of source files: $i$ ) the main subroutine suspect2.f together with a separate routine twoloophiggs.f for the two-loop radiative corrections in the Higgs sector, $i i$ ) a separate calling routine file suspect2_call.f, and $i i i$ ) two possible input files suspect2. in (in the original SuSpect format) or suspect2_lha. in (in the SLHA format). Any choice and option is driven either from one of the two input files [which is sufficient and convenient when dealing with a few model points] or alternatively directly from the suspect2_call.f file, which also provides examples of calls for different model choices with all the necessary features [this option being useful when interfacing with other routines or when performing scans over the parameter space]. The program has several flags which allow to select the model to be studied and its input parameters, the level of accuracy of the algorithm [e.g. the iterations for the RGEs and the convergence of the EWSB], the level of approximation in the calculation of the various (s)particle masses [e.g. inclusion or not of $\mathrm{RC}$ ]. Besides the fact that it is flexible, the code is self-contained [the default version includes all routines needed for the calculation], rather fast [thus allowing large scans of the parameter space] and can be easily linked to other routines or Monte-Carlo generators [e.g. to calculate branching ratios, cross sections, relic densities]. All results, including comments when useful and some theoretical and experimental constraints, are found in the output file suspect2.out (in the original SuSpect format by default) or alternatively in the output file suspect2_lha.out, which are created at any run of the program. It is hoped that the code may be readily usable even without much prior knowledge of the MSSM.

This "users' manual" for the program, is organized as follows. In section 2, we briefly discuss the main ingredients of the unconstrained and phenomenological MSSMs as well as the constrained models mSUGRA, AMSB and GMSB, to set the notations and conventions used in the program. In section 3, we summarize the procedure for the calculation of the (s)particle spectrum: the soft SUSY-breaking terms [including the treatment of the input, the RG evolution and the implementation of EWSB], the physical particle masses [summarizing our conventions for the sfermion, gaugino and Higgs sectors]. We also discuss the theoretical [CCB, UFB, fine-tuning] and experimental [electroweak precision observables, muon $g-2, b \rightarrow s \gamma$ branching fraction] constraints which can be imposed on the spectra, and how these are implemented in the code. In section 4, we summarize the basic practical facts about the program and discuss the content of the input and output files with the possible choices; we then make a brief comparison with other existing codes, discuss the interface with other programs and how the program is maintained on the web. A conclusion will be given in section 5 . In the 


\section{The constrained and unconstrained MSSMs}

In this section, we summarize the basic assumptions which define the MSSM and the various constraints which can be imposed on it. This will also set the notations and conventions used in the program. We mainly focus on the unconstrained MSSM, the phenomenological MSSM with 22 free parameters, as well as on constrained models such as the minimal Supergravity (mSUGRA), anomaly mediated (AMSB) and gauge mediated (GMSB) supersymmetry breaking models.

\subsection{The unconstrained MSSM}

The unconstrained MSSM is defined usually by the following four basic assumptions [36]:

(a) Minimal gauge group: the MSSM is based on the group $\mathrm{SU}(3)_{\mathrm{C}} \times \mathrm{SU}(2)_{\mathrm{L}} \times \mathrm{U}(1)_{\mathrm{Y}}$, i.e. the SM symmetry. SUSY implies then that the spin-1 gauge bosons and their spin- $1 / 2$ partners, the gauginos [bino $\tilde{B}$, winos $\tilde{W}_{1-3}$ and gluinos $\tilde{G}_{1-8}$ ], are in vector supermultiplets.

(b) Minimal particle content: there are only three generations of spin- $1 / 2$ quarks and leptons as in the SM. The left- and right-handed chiral fields belong to chiral superfields together with their spin-0 SUSY partners, the squarks and sleptons: $\hat{Q}, \hat{u}_{R}, \hat{d}_{R}, \hat{L}, \hat{l}_{R}$. In addition, two chiral superfields $\hat{H}_{d}, \hat{H}_{u}$ with respective hypercharges -1 and +1 for the cancellation of chiral anomalies, are needed. Their scalar components, $H_{d}$ and $H_{u}$, give separately masses to the isospin $+1 / 2$ and $-1 / 2$ fermions and lead to five Higgs particles: two $\mathrm{CP}-$ even $h, H$ bosons, a pseudoscalar $A$ boson and two charged $H^{ \pm}$bosons. Their spin- $1 / 2$ superpartners, the higgsinos, will mix with the winos and the bino, to give the "ino" mass eigenstates: the two charginos $\chi_{1,2}^{ \pm}$and the four neutralinos $\chi_{1,2,3,4}^{0}$.

(c) Minimal Yukawa interactions and R-parity conservation: to enforce lepton and baryon number conservation, a discrete and multiplicative symmetry called $\mathrm{R}$-parity is imposed. It is defined by $R_{p}=(-1)^{2 s+3 B+L}$, where $\mathrm{L}$ and $\mathrm{B}$ are the lepton and baryon numbers and $s$ is the spin quantum number. The $\mathrm{R}$-parity quantum numbers are then $R_{p}=+1$ for the ordinary particles [fermions, gauge and Higgs bosons], and $R_{p}=-1$ for their supersymmetric partners. In practice, the conservation of $R$-parity has important consequences: the SUSY particles are always produced in pairs, in their decay products there is always an odd number of SUSY particles, and the lightest SUSY particle (LSP) is absolutely stable.

The three conditions listed above are sufficient to completely determine a globally supersymmetric Lagrangian. The kinetic part of the Lagrangian is obtained by generalizing the notion of covariant derivative to the SUSY case. The most general superpotential, compatible with gauge invariance, renormalizability and $\mathrm{R}$-parity conservation is written as:

$$
W=\sum_{i, j=g e n}-Y_{i j}^{u} \hat{u}_{R i} \hat{H}_{u} \cdot \hat{Q}_{j}+Y_{i j}^{d} \hat{d}_{R i} \hat{H}_{d} \cdot \hat{Q}_{j}+Y_{i j}^{l} \hat{l}_{R i} \hat{H}_{u} \cdot \hat{L}_{j}+\mu \hat{H}_{u} \cdot \hat{H}_{d}
$$

The product between $\mathrm{SU}(2)_{\mathrm{L}}$ doublets reads $H \cdot Q \equiv \epsilon_{a b} H^{a} Q^{b}$ where $a, b$ are $\mathrm{SU}(2)_{\mathrm{L}}$ indices 
and $\epsilon_{12}=1=-\epsilon_{21}$, and $Y_{i j}^{u, d, l}$ denote the Yukawa couplings among generations. The first three terms in the previous expression are nothing else but a superspace generalization of the Yukawa interaction in the SM, while the last term is a globally supersymmetric Higgs mass term. The supersymmetric part of the tree-level potential $V_{\text {tree }}$ is the sum of the so-called F- and D-terms [37], where the F-terms come from the superpotential through derivatives with respect to all scalar fields $\phi_{a}, V_{F}=\sum_{a}\left|W^{a}\right|^{2}$ with $W^{a}=\partial W / \partial \phi_{a}$, and the D-terms corresponding to the $\mathrm{U}(1)_{\mathrm{Y}}, \mathrm{SU}(2)_{\mathrm{L}}$, and $\mathrm{SU}(3)_{\mathrm{C}}$ gauge symmetries are given by $V_{D}=\frac{1}{2} \sum_{i=1}^{3}\left(\sum_{a} g_{i} \phi_{a}^{*} T^{i} \phi_{a}\right)^{2}$ with $T^{i}$ and $g_{i}$ being the generators and the coupling constants of the corresponding gauge groups.

(d) Minimal set of soft SUSY-breaking terms: to break Supersymmetry, while preventing the reappearance of the quadratic divergences [soft breaking], one adds to the supersymmetric Lagrangian a set of terms which explicitly but softly break SUSY [38]:

- Mass terms for the gluinos, winos and binos:

$$
-\mathcal{L}_{\text {gaugino }}=\frac{1}{2}\left[M_{1} \tilde{B} \tilde{B}+M_{2} \sum_{a=1}^{3} \tilde{W}^{a} \tilde{W}_{a}+M_{3} \sum_{a=1}^{8} \tilde{G}^{a} \tilde{G}_{a}+\text { h.c. }\right]
$$

- Mass terms for the scalar fermions:

$$
-\mathcal{L}_{\text {sfermions }}=\sum_{i=g e n} m_{\tilde{Q} i}^{2} \tilde{Q}_{i}^{\dagger} \tilde{Q}_{i}+m_{\tilde{L} i}^{2} \tilde{L}_{i}^{\dagger} \tilde{L}_{i}+m_{\tilde{u} i}^{2}\left|\tilde{u}_{R_{i}}\right|^{2}+m_{\tilde{d} i}^{2}\left|\tilde{d}_{R_{i}}\right|^{2}+m_{\tilde{l} i}^{2}\left|\tilde{l}_{R_{i}}\right|^{2}
$$

- Mass and bilinear terms for the Higgs bosons:

$$
-\mathcal{L}_{\text {Higgs }}=m_{H_{u}}^{2} H_{u}^{\dagger} H_{u}+m_{H_{d}}^{2} H_{d}^{\dagger} H_{d}+B \mu\left(H_{u} . H_{d}+\text { h.c. }\right)
$$

- Trilinear couplings between sfermions and Higgs bosons

$$
-\mathcal{L}_{\text {tril. }}=\sum_{i, j=g e n}\left[A_{i j}^{u} Y_{i j}^{u} \tilde{u}_{R_{i}} H_{u} \cdot \tilde{Q}_{j}+A_{i j}^{d} Y_{i j}^{d} \tilde{d}_{R_{i}} H_{d} \cdot \tilde{Q}_{j}+A_{i j}^{l} Y_{i j}^{l} \tilde{l}_{R_{i}} H_{u} \cdot \tilde{L}_{j}+\text { h.c. }\right]
$$

The soft SUSY-breaking scalar potential is the sum of the three last terms:

$$
V_{\text {soft }}=-\mathcal{L}_{\text {sfermions }}-\mathcal{L}_{\text {Higgs }}-\mathcal{L}_{\text {tril }}
$$

Up to now, no constraint is applied to this Lagrangian, although for generic values of the parameters, it might lead to severe phenomenological problems such as flavor changing neutral currents [FCNC] and unacceptable amount of additional CP-violation [39], color and/or charge breaking minima [40], an incorrect value of the $Z$ boson mass, etc... The MSSM defined by the four hypotheses $(a)-(d)$ above, will be called the unconstrained MSSM. 


\subsection{The "phenomenological" MSSM}

In the unconstrained MSSM, and in the general case where one allows for intergenerational mixing and complex phases, the soft SUSY breaking terms will introduce a huge number (105) of unknown parameters, in addition to the 19 parameters of the SM [7]. This large number of free parameters makes any phenomenological analysis in the general MSSM very complicated as mentioned previously. In addition, many "generic" sets of these parameters are excluded by the severe phenomenological constraints discussed above. A phenomenologically viable MSSM can be defined by making the following three assumptions: $(i)$ All the soft SUSY-breaking parameters are real and therefore there is no new source of $\mathrm{CP}$-violation generated, in addition to the one from the CKM matrix. (ii) The matrices for the sfermion masses and for the trilinear couplings are all diagonal, implying the absence of FCNCs at the tree-level. (iii) First and second sfermion generation universality at low energy to cope with the severe constraints from $K^{0}-\bar{K}^{0}$ mixing, etc [this is also motivated by the fact that one can neglect for simplicity all the masses of the first and second generation fermions which are too small to have any effect on the running of the SUSY-breaking parameters].

Making these three assumptions will lead to 22 input parameters only:

$\tan \beta$ : the ratio of the vevs of the two-Higgs doublet fields.

$m_{H_{u}}^{2}, m_{H_{d}}^{2}$ : the Higgs mass parameters squared.

$M_{1}, M_{2}, M_{3}$ : the bino, wino and gluino mass parameters.

$m_{\tilde{q}}, m_{\tilde{u}_{R}}, m_{\tilde{d}_{R}}, m_{\tilde{l}}, m_{\tilde{e}_{R}}:$ the first/second generation sfermion mass parameters.

$m_{\tilde{Q}}, m_{\tilde{t}_{R}}, m_{\tilde{b}_{R}}, m_{\tilde{L}}, m_{\tilde{\tau}_{R}}$ : the third generation sfermion mass parameters.

$A_{u}, A_{d}, A_{e}$ : the first/second generation trilinear couplings.

$A_{t}, A_{b}, A_{\tau}$ : the third generation trilinear couplings.

Two remarks can be made at this stage: $(i)$ The Higgs-higgsino (supersymmetric) mass parameter $|\mu|$ (up to a sign) and the soft SUSY-breaking bilinear Higgs term $B$ are determined, given the above parameters, through the electroweak symmetry breaking conditions as will be discussed later. Alternatively, one can trade the values of $m_{H_{u}}^{2}$ and $m_{H_{d}}^{2}$ with the "more physical" pseudoscalar Higgs boson mass $M_{A}$ and parameter $\mu$ [such an alternative choice is explicitly possible in SuSpect by appropriate setting of the input parameters]. (ii) Since the trilinear sfermion couplings will be always multiplied by the fermion masses, they are important only in the case of the third generation. However, there are a few (low scale) situations, such as the muon $(g-2)$ and the neutralino-nucleon scattering for direct Dark Matter searches, where they will play a role. We thus consider them as input.

Such a model, with this relatively moderate number of parameters [especially that, in general, only a small subset contributes dominantly (i.e. at tree-level) when one looks at a given sector of the model] has much more predictability and is much easier to investigate phenomenologically, compared to the unconstrained MSSM. We will refer to this 22 free input parameters model as the "phenomenological" MSSM or pMSSM [4]. 


\subsection{The mSUGRA model}

Almost all problems of the general or unconstrained MSSM are solved at once if the soft SUSY-breaking parameters obey a set of universal boundary conditions at the GUT scale. If one takes these parameters to be real, this solves all potential problems with $\mathrm{CP}$ violation as well. The underlying assumption is that SUSY-breaking occurs in a hidden sector which communicates with the visible sector only through gravitational-strength interactions, as specified by Supergravity. Universal soft breaking terms then emerge if these Supergravity interactions are "flavor-blind" [like ordinary gravitational interactions]. This is assumed to be the case in the constrained MSSM or minimal Supergravity (mSUGRA) model [8].

Besides the unification of the gauge coupling constants $g_{1,2,3}$ of the $\mathrm{U}(1), \mathrm{SU}(2)$ and $\mathrm{SU}(3)$ groups, which is well verified given the experimental results from LEP1 [41] and which can be viewed as fixing the Grand Unification scale $M_{\mathrm{GUT}} \sim 2 \cdot 10^{16} \mathrm{GeV}$ [42], the unification conditions in mSUGRA, are as follows:

- Unification of the gaugino [bino, wino and gluino] masses:

$$
M_{1}\left(M_{\mathrm{GUT}}\right)=M_{2}\left(M_{\mathrm{GUT}}\right)=M_{3}\left(M_{\mathrm{GUT}}\right) \equiv m_{1 / 2}
$$

- Universal scalar [i.e. sfermion and Higgs boson] masses [i is the generation index]:

$$
\begin{aligned}
M_{\tilde{Q}_{i}}\left(M_{\mathrm{GUT}}\right) & =M_{\tilde{u}_{R i}}\left(M_{\mathrm{GUT}}\right)=M_{\tilde{d}_{R i}}\left(M_{\mathrm{GUT}}\right)=M_{\tilde{L}_{i}}\left(M_{\mathrm{GUT}}\right)=M_{\tilde{l}_{R i}}\left(M_{\mathrm{GUT}}\right) \\
& =M_{H_{u}}\left(M_{\mathrm{GUT}}\right)=M_{H_{d}}\left(M_{\mathrm{GUT}}\right) \equiv m_{0}
\end{aligned}
$$

- Universal trilinear couplings:

$$
A_{i j}^{u}\left(M_{\mathrm{GUT}}\right)=A_{i j}^{d}\left(M_{\mathrm{GUT}}\right)=A_{i j}^{l}\left(M_{\mathrm{GUT}}\right) \equiv A_{0} \delta_{i j}
$$

Besides the three parameters $m_{1 / 2}, m_{0}$ and $A_{0}$, the supersymmetric sector is described at the GUT scale by the bilinear coupling $B$ and the supersymmetric Higgs(ino) mass parameter $\mu$. However, one has to require that EWSB takes place at some low energy scale. This results in two necessary minimization conditions of the two-Higgs doublet scalar potential which, at the tree-level, has the form [to have a more precise description, one-loop corrections to the scalar potential have to be included, as will be discussed later]:

$$
\begin{aligned}
V_{\mathrm{Higgs}} & =\bar{m}_{1}^{2} H_{d}^{\dagger} H_{d}+\bar{m}_{2}^{2} H_{u}^{\dagger} H_{u}+\bar{m}_{3}^{2}\left(H_{u} \cdot H_{d}+\text { h.c. }\right) \\
& +\frac{g_{1}^{2}+g_{2}^{2}}{8}\left(H_{d}^{\dagger} H_{d}-H_{u}^{\dagger} H_{u}\right)^{2}+\frac{g_{2}^{2}}{2}\left(H_{d}^{\dagger} H_{u}\right)\left(H_{u}^{\dagger} H_{d}\right)
\end{aligned}
$$

where we have used the usual short-hand notation: $\bar{m}_{1}^{2}=m_{H_{d}}^{2}+\mu^{2}, \bar{m}_{2}^{2}=m_{H_{u}}^{2}+\mu^{2}$, $\bar{m}_{3}^{2}=B \mu$ and the SU(2) invariant product of the two doublets $\phi_{1} \cdot \phi_{2}=\phi_{1}^{1} \phi_{2}^{2}-\phi_{1}^{2} \phi_{2}^{1}$. The two minimization equations $\partial V_{\text {Higgs }} / \partial H_{d}^{0}=\partial V_{\text {Higgs }} / \partial H_{u}^{0}=0$ can be solved for $\mu^{2}$ and $B \mu$ :

$$
\begin{aligned}
\mu^{2} & =\frac{1}{2}\left[\tan 2 \beta\left(m_{H_{u}}^{2} \tan \beta-m_{H_{d}}^{2} \cot \beta\right)-M_{Z}^{2}\right] \\
B \mu & =\frac{1}{2} \sin 2 \beta\left[m_{H_{u}}^{2}+m_{H_{d}}^{2}+2 \mu^{2}\right]
\end{aligned}
$$


(which are of course valid as well in the more general unconstrained MSSM). Here, $M_{Z}^{2}=$ $\left(g_{1}^{2}+g_{2}^{2}\right) \cdot\left(v_{u}^{2}+v_{d}^{2}\right) / 4$ and $\tan \beta=v_{u} / v_{d}$ is defined in terms of the vacuum expectation values of the two neutral Higgs fields. Consistent EWSB is only possible if eq. (11) gives a positive value of $\mu^{2}$. The sign of $\mu$ is not determined. Therefore, in this model, one is left with only four continuous free parameters, and an unknown sign:

$$
\tan \beta, m_{1 / 2}, m_{0}, A_{0}, \operatorname{sign}(\mu) .
$$

All the soft SUSY breaking parameters at the weak scale are then obtained through Renormalization Group Equations.

\subsection{The AMSB model}

In mSUGRA, Supersymmetry is broken in a hidden sector and the breaking is transmitted to the visible sector by gravitational interactions. In Anomaly Mediated Supersymmetry Breaking models, the SUSY-breaking occurs also in a hidden sector, but it is transmitted to the visible sector by the super-Weyl anomaly $[9,10]$. The gaugino, scalar masses and trilinear couplings are then simply related to the scale dependence of the gauge and matter kinetic functions. This leads to soft SUSY-breaking scalar masses for the first two generation sfermions that are almost diagonal [when the small Yukawa couplings are neglected].

In terms of the gravitino mass $m_{3 / 2}$ [which is much larger than the gaugino and squark masses, a cosmologically appealing feature], the $\beta$ functions for the gauge and Yukawa couplings $g_{a}$ and $Y_{i}$, and the anomalous dimensions $\gamma_{i}$ of the chiral superfields, the soft SUSY breaking terms are given by:

$$
\begin{aligned}
M_{a} & =\frac{\beta_{g_{a}}}{g_{a}} m_{3 / 2} \\
A_{i} & =\frac{\beta_{Y_{i}}}{Y_{i}} m_{3 / 2} \\
m_{i}^{2} & =-\frac{1}{4}\left(\Sigma_{a} \frac{\partial \gamma_{i}}{\partial g_{a}} \beta_{g_{a}}+\Sigma_{k} \frac{\partial \gamma_{i}}{\partial Y_{k}} \beta_{Y_{k}}\right) m_{3 / 2}^{2}
\end{aligned}
$$

These equations are RG invariant and thus valid at any scale and make the model highly predictive. The additional parameters, $\mu^{2}$ and $B$ are obtained as usual by requiring the correct breaking of the electroweak symmetry. One then has, in principle, only three input parameters $m_{3 / 2}, \tan \beta$ and $\operatorname{sign}(\mu)$. However, this rather simple picture is spoiled by the fact that the anomaly mediated contribution to the slepton scalar masses squared is negative and the sleptons are in general tachyonic. This problem can be cured by adding a positive non-anomaly mediated contribution to the soft masses. The simplest phenomenological way of parameterizing the non-anomaly contribution is to add a common mass parameter $m_{0}$ at the GUT scale, which would be then an additional input parameter to all the (squared) scalar masses. However in the general case, the non-anomaly mediated contribution might 
be different for different scalar masses and depend on the specific model which has been chosen. One should then write a general non-anomalous contribution at the GUT scale for each scalar mass squared:

$$
m_{\tilde{S}_{i}}^{2}=c_{S_{i}} m_{0}^{2}-\frac{1}{4}\left(\Sigma_{a} \frac{\partial \gamma_{i}}{\partial g_{a}} \beta_{g_{a}}+\Sigma_{k} \frac{\partial \gamma_{i}}{\partial Y_{k}} \beta_{Y_{k}}\right) m_{3 / 2}^{2}+\text { D terms. }
$$

where the coefficients $c_{S_{i}}$ depend on the considered model.

A few examples of models with different non-anomalous contributions are:

- The minimal anomaly mediated supersymmetry breaking model with a universal $m_{0}[43]$ :

$$
c_{Q}=c_{u_{R}}=c_{d_{R}}=c_{L}=c_{e_{R}}=c_{H_{u}}=c_{H_{d}}=1
$$

- The gaugino assisted AMSB model where one assumes that gauge and gaugino fields reside in the bulk of an extra dimension [44]:

$$
c_{Q}=21 / 10, c_{u_{R}}=8 / 5, c_{d_{R}}=7 / 5, c_{L}=9 / 10, c_{e}=3 / 5, c_{H_{u}}=9 / 10 c_{H_{d}}
$$

- Models where an extra U(1) factor is added; a particular scenario is interesting phenomenologically since it leads to a light top squark [45]:

$$
c_{Q}=3, c_{u_{R}}=c_{d_{R}}=-1, c_{L}=c_{e}=1, c_{H_{u}}=c_{H_{d}}=-2
$$

A simple way to account for all the different models is to add to the three continuous and one discrete original basic parameters, the set of coefficients $c_{S_{i}}$ as input to specify, and therefore one would have the set of input parameters:

$$
m_{0}, m_{3 / 2}, \tan \beta, \operatorname{sign}(\mu) \text { and } c_{S_{i}}
$$

This is the approach that we will follow in the program.

\subsection{The GMSB model}

In Gauge Mediated Supersymmetry Breaking models, SUSY-breaking is transmitted to the MSSM fields via the SM gauge interactions. In the original scenario [46], the model consists of three distinct sectors: a secluded sector where SUSY is broken, a "messenger" sector containing a singlet field and messenger fields with $\mathrm{SU}(3)_{\mathrm{c}} \times \mathrm{SU}(2)_{\mathrm{L}} \times \mathrm{U}(1)_{\mathrm{Y}}$ quantum numbers, and a sector containing the fields of the MSSM. Another possibility, the so-called "direct gauge mediation" [47] has only two sectors: one which is responsible for the SUSY breaking and contains the messenger fields, and another sector consisting of the MSSM fields. In both cases, the soft SUSY-breaking masses for the gauginos and squared masses for the sfermions arise, respectively, from one-loop and two-loop diagrams involving the exchange of the messenger fields, while the trilinear Higgs-sfermion-sfermion couplings can be taken 
to be negligibly small at the messenger scale since they are [and not their square as for the sfermion masses] generated by two-loop gauge interactions. This allows an automatic and natural suppression of FCNC and CP-violation; for a review see, Ref. [12].

In the GMSB models that we will consider, the source of SUSY breaking is parameterized by an $\mathrm{SU}(3)_{\mathrm{C}} \times \mathrm{SU}(2)_{\mathrm{L}} \times \mathrm{U}(1)_{\mathrm{Y}}$ gauge-singlet chiral superfield $\hat{S}$ whose scalar and auxiliary components acquire vacuum expectation values denoted by $S$ and $F_{S}$, respectively. We assume $n_{\hat{q}}$ pairs of $\hat{q}, \hat{\bar{q}}$ quark-like [resp. $n_{\hat{l}}$ pairs of $\hat{l}, \hat{\bar{l}}$ lepton-like] messenger superfields transforming as $\left(3,1,-\frac{1}{3}\right),\left(\overline{3}, 1, \frac{1}{3}\right)\left[\right.$ resp. $\left.\left(1,2, \frac{1}{2}\right),\left(1,2,-\frac{1}{2}\right)\right]$ under $\mathrm{SU}(3)_{\mathrm{C}} \times \mathrm{SU}(2)_{\mathrm{L}} \times \mathrm{U}(1)_{\mathrm{Y}}$ and coupled to $\hat{S}$ through a superpotential of the form $\lambda \hat{S} \hat{q} \hat{\bar{q}}+\lambda \hat{S} \hat{\bar{l}} \hat{\bar{l}}$. Soft SUSY-breaking parameters are then generated at the messenger scale $M_{\text {mes }}=\lambda S$,

$$
\begin{aligned}
M_{G}\left(M_{\mathrm{mes}}\right) & =\frac{\alpha_{G}\left(M_{\mathrm{mes}}\right)}{4 \pi} \Lambda g\left(\frac{\Lambda}{M_{\mathrm{mes}}}\right) \sum_{m} N_{R}^{G}(m) \\
m_{s}^{2}\left(M_{\mathrm{mes}}\right) & =2 \Lambda^{2} f\left(\frac{\Lambda}{M_{\mathrm{mes}}}\right) \sum_{m, G}\left[\frac{\alpha_{G}\left(M_{\mathrm{mes}}\right)}{4 \pi}\right]^{2} N_{R}^{G}(m) C_{R}^{G}(s) \\
A_{f}\left(M_{\mathrm{mes}}\right) & \simeq 0
\end{aligned}
$$

where $\Lambda=F_{S} / S, G=\mathrm{U}(1), \mathrm{SU}(2), \mathrm{SU}(3), m$ labels the messengers and $s$ runs over the Higgs doublets as well as the left-handed doublets and right-handed singlets of squarks and sleptons. The one- and two-loop functions $g$ and $f$ are given by $\left[\mathrm{Li}_{2}\right.$ is the Spence function]:

$$
\begin{aligned}
& g(x)=\frac{1}{x^{2}}[(1+x) \log (1+x)+(1-x) \log (1-x)] \\
& f(x)=\frac{1+x}{x^{2}}\left[\log (1+x)-2 \operatorname{Li}_{2}\left(\frac{x}{1+x}\right)+\frac{1}{2} \operatorname{Li}_{2}\left(\frac{2 x}{1+x}\right)\right]+(x \leftrightarrow-x)
\end{aligned}
$$

Defining the Dynkin index $N_{R}^{G}$ by

$$
\operatorname{Tr}\left(T_{R}^{a} T_{R}^{b}\right)=\frac{N_{R}^{G}}{2} \delta^{a b}
$$

for non-abelian groups, and $N^{U(1)_{Y}}=(6 / 5) Y^{2}$ where $Y \equiv Q_{\mathrm{EM}}-T_{3}$, one has (see eq.(191))

$$
\begin{aligned}
\sum_{m} N_{R}^{U(1)_{Y}}(m) & =\frac{1}{5}\left(2 n_{\hat{q}}+3 n_{\hat{l}}\right) \\
\sum_{m} N_{R}^{S U(2)_{L}}(m) & =n_{\hat{l}} \\
\sum_{m} N_{R}^{S U(3)_{c}}(m) & =n_{\hat{q}}
\end{aligned}
$$

With the Casimir invariant $C_{\mathbf{N}}^{G}$ given by

$$
\Sigma_{a} T_{\mathbf{N}}^{a} T_{\mathbf{N}}^{a}=C_{\mathbf{N}}^{S U(N)} \mathbf{1}=\frac{N^{2}-1}{2 N} \mathbf{1}
$$

for the $\mathbf{N}$ of $S U(N)$, and $C^{U(1)_{Y}}=(3 / 5) Y^{2}$, one finds for

$$
\mathcal{N C}(s) \equiv \sum_{m, G}\left[\frac{\alpha_{G}\left(M_{\mathrm{mes}}\right)}{4 \pi}\right]^{2} N_{R}^{G}(m) C_{R}^{G}(s)
$$


(see eq.(201) ) the following values:

$$
\begin{aligned}
\mathcal{N C}(\tilde{Q}) & =\frac{1}{16 \pi^{2}}\left[\left(\frac{n_{\hat{l}}}{100}+\frac{n_{\hat{q}}}{150}\right) \alpha_{1}^{2}+\frac{3 n_{\hat{l}}}{4} \alpha_{2}^{2}+\frac{4 n_{\hat{q}}}{3} \alpha_{3}^{2}\right] \\
\mathcal{N C}(\tilde{U}) & =\frac{1}{16 \pi^{2}}\left[\left(\frac{4 n_{\hat{l}}}{25}+\frac{8 n_{\hat{q}}}{75}\right) \alpha_{1}^{2}+\frac{4 n_{\hat{q}}}{3} \alpha_{3}^{2}\right] \\
\mathcal{N C}(\tilde{D}) & =\frac{1}{16 \pi^{2}}\left[\left(\frac{n_{\hat{l}}}{25}+\frac{2 n_{\hat{q}}}{75}\right) \alpha_{1}^{2}+\frac{4 n_{\hat{q}}}{3} \alpha_{3}^{2}\right] \\
\mathcal{N C}(\tilde{L}) & =\frac{1}{16 \pi^{2}}\left[\left(\frac{9 n_{\hat{l}}}{100}+\frac{3 n_{\hat{q}}}{50}\right) \alpha_{1}^{2}+\frac{3 n_{\hat{l}}}{4} \alpha_{2}^{2}\right] \\
\mathcal{N C}(\tilde{E}) & =\frac{1}{16 \pi^{2}}\left[\left(\frac{9 n_{\hat{l}}}{25}+\frac{6 n_{\hat{q}}}{25}\right) \alpha_{1}^{2}\right] \\
\mathcal{N C}\left(\tilde{H}_{u}\right) & =\mathcal{N C}\left(\tilde{H}_{d}\right)=\mathcal{N C}(\tilde{L})
\end{aligned}
$$

The freedom in choosing independently the number of $n_{\hat{q}}$ and $n_{\hat{l}}$ messengers allows to study various model configurations: for instance when the messengers are assumed to form complete representations of some grand unification group (e.g. $\mathbf{5}+\overline{\mathbf{5}}$ of $\mathrm{SU}(5))$ where $n_{\hat{q}}=n_{\hat{l}}$, or when they transform under larger unification group factors with some extra discrete symmetries where typically $n_{\hat{q}} \neq n_{\hat{l}}$ [48]. [When $n_{\hat{q}}=n_{\hat{l}}=1$ one retrieves the minimal model [46]. In this case the gaugino masses have the same relative values as if they were unified at $M_{\mathrm{GUT}}$ despite the fact the boundary conditions are set at $M_{\text {mes }}$ and that scalar masses are flavor independent. Furthermore when $\Lambda / M_{\text {mes }} \ll 1$, one has $f(x) \simeq g(x) \simeq 1$.] In addition, some constraints are in general needed in order to have a viable spectrum, for instance: $\Lambda / M_{\text {mes }}<1$ to avoid negative mass squared for bosonic members of the messenger scale and $\Lambda / M_{\text {mes }} \lesssim 0.9$ to avoid too much fine-tuning in EWSB. Note also that $n_{\hat{q}}>n_{\hat{l}}$ improves the fine-tuning issue [49].

Once the boundary conditions are set at $M_{\text {mes }}$, the low energy parameters are obtained via the usual RGEs and the proper breaking of the EW symmetry is required.

Therefore, in the GMSB model that we are considering, there are six input parameters

$$
\tan \beta, \operatorname{sign}(\mu), M_{\text {mes }}, \Lambda, n_{\hat{q}}, n_{\hat{l}}
$$

In addition, one has to include as input the mass of the gravitino $\tilde{G}$ which, in this case is the lightest SUSY particle. This mass, $m_{\tilde{G}}=F /\left(\sqrt{3} M_{P}\right)$ with $M_{P}$ the reduced Planck mass, will depend on an additional free parameter $F$ which parameterizes the scale of the full SUSY breaking and whose typical size is of $\mathcal{O}\left(F_{S}\right)$ in direct mediation and much larger in secluded mediation. The choice of this parameter, which plays a role only for the lifetime of the next-to-lightest SUSY particle, is left to the user.

\subsection{Non-universality models}

mSUGRA, AMSB and GMSB are well defined models of which the possible phenomenological consequences and experimental signatures have been widely studied in the literature. 
However, in the absence of a truly fundamental description of SUSY-breaking, none of these models should be considered as THE compelling model. Unknown physics at the Planck and/or GUT scales renders some of the basic assumptions inherent to the above scenarii rather uncertain: deviations from mSUGRA universality conditions at the high scale are generally expected in non-minimal Supergravity [50], or in superstring [51] settings while deviations from the initial AMSB scenario can be expected, due to extra anomaly contributions coming from the underlying fundamental theory [10] and deviations from minimal GMSB can occur due for instance to the breaking of symmetries which protected direct coupling between messenger and MSSM matter fields.

To be on the safe side from the experimental point of view, it is therefore wiser to allow for a departure from these models, and to study the phenomenological implications of relaxing some defining assumptions. However, it is often desirable to limit the number of extra free parameters, in order to retain a reasonable amount of predictability when attempting detailed investigations of possible signals of SUSY. Therefore, it is more interesting to relax only one [or a few] assumption[s] at a time and study the phenomenological implications. Of course, since there are many possible directions, this would lead to several intermediate MSSMs between these constrained models and the phenomenological MSSM with 22 free parameters discussed in section 2.2.

Taking the most studied mSUGRA as a reference model, we briefly discuss here some model cases where the universality conditions are naturally violated:

i) non unification of the soft SUSY-breaking gaugino mass terms:

$$
M_{1}\left(M_{U}\right) \neq M_{2}\left(M_{U}\right) \neq M_{3}\left(M_{U}\right)
$$

This occurs for instance in Superstring motivated models in which the SUSY breaking is moduli dominated such as in the O-I and O-II models [52], or in extra dimensional SUSYGUT models in which the additional dimensions lead to the breaking of the large gauge symmetry and/or supersymmetry or in SUSY models where the breaking occurs through a non SU(5) singlet $F$ term; see Ref. [53] for phenomenology oriented discussions.

ii) mSUGRA with non-unification of the two first and third generation scalar masses:

$$
m_{0 \tilde{Q}}=m_{0 \tilde{L}} \cdots \neq m_{0 \tilde{q}}=m_{0 \tilde{l}} \cdots
$$

This occurs in models where the soft SUSY-breaking scalar masses at the GUT scale are influenced by the fermion Yukawa couplings. This is the case for the so-called inverted mass hierarchy models [54] where the scalar mass terms of the first two generations can be very heavy $\mathcal{O}(10 \mathrm{TeV})$, while those of the third generation sfermions and the Higgs bosons are rather light, solving thus the SUSY flavor and CP problems, which are related to the first two generations, while still satisfying naturalness constraints.

iii) mSUGRA-like models, but with non-universality of the sfermion and Higgs boson scalar masses [keeping a priori universal gaugino masses and trilinear soft breaking couplings] . In a model-independent context, one can in principle consider any non-universality 
configuration which does not violate important constraints from FCNC, etc... Interestingly, such configurations occur naturally in models where the grand unification group has a rank strictly greater than 4 [55]. Starting from universal soft scalar masses, non-universality effects occur at intermediate scales, related to the spontaneous symmetry breaking down to the standard model gauge group, via D-term contributions corresponding to the extra U(1) symmetries [56]. For instance, in SO(10) SUSY GUT models with universal boundary conditions, and assuming that the GUT group breaks directly to the SM group at the GUT scale, one is lead to patterns of the form $[56,57]$,

$$
m_{\tilde{Q}}=m_{\tilde{e}_{R}}=m_{\tilde{u}_{R}} \neq m_{\tilde{d}_{R}}=m_{\tilde{L}} \neq M_{H_{u}} \neq M_{H_{d}}
$$

where there are actually three free mass parameters, two of which can be taken as the initial common soft masses for the sfermion and Higgs $\mathrm{SO}(10)$ multiplets, the third being the D-term contribution associated to the reduction of the group rank.

In the present SuSpect version such specific SUSY GUT models are not yet available ${ }^{1}$, but one can still have a reasonable estimate of the non-universality effects by using the pMSSM option. Alternatively, one can also use the SuSpect option which allows to disconnect the Higgs sector from the sfermionic by introducing two additional input parameters: the pseudoscalar Higgs boson mass $M_{A}$ and the higgsino mass parameter $\mu$ [which have a more direct "physical" interpretation than the scalar mass terms $\left.M_{H_{u}}, M_{H_{d}}\right]$. This allows to perform more general phenomenological or experimental analyses; c.f. some LEP and LHC Higgs analyses [58] or some recent Dark Matter studies [59].

iv) Partially unified models where one relaxes one or a few parameters to fit some collider zoo event or to analyze a phenomenological situation which introduces new features. This is the case, for instance, for the light top squark scenario which can be set by hand to discuss some theoretical issues [such as baryogenesis in the MSSM [60] for instance] or phenomenological situations [such as new decay or production modes of top squarks [61] for instance] .

An easy and practical way to implement these various non-unified or partially unified scenarii, is to allow for the possibility of choosing all the soft SUSY-breaking parameters listed above for the phenomenological MSSM of section 2.2 [the 22 parameters except for $\tan \beta]$ at the high-energy or GUT scale, with the boundary conditions set by hand and chosen at will. One can even chose the scale at which the boundary conditions are set to account for intermediate scales. If this scale is the electroweak symmetry breaking scale, then we have simply the MSSM with the soft SUSY-breaking parameters defined at the low energy scale, i.e. the phenomenological MSSM. All these options are provided by our code.

\footnotetext{
${ }^{1}$ These would require modified RGEs at least for some sectors, inclusion of the effects of new heavy fields (right-handed (s)neutrinos for instance), of threshold effects corresponding to the various scales of successive symmetry breaking down to the SM, etc...
} 


\section{The Particle Spectrum Calculation with Suspect}

In this section, we discuss our procedure for calculating the SUSY and Higgs particle spectrum. We will take as example the sophisticated cases of the constrained MSSMs with universal boundary conditions at the high scale, mSUGRA AMSB and GMSB, where all ingredients included in the SuSpect algorithm are present: RGEs, radiative EWSB and calculation of the physical particle masses. We first describe the general algorithm, then discuss the calculation of the soft SUSY-breaking terms, the determination of the particle masses, the various theoretical and phenomenological tests that we impose on the model parameters.

\subsection{General algorithm}

As mentioned previously, there are three main steps for the calculation of the supersymmetric particle spectrum in constrained MSSMs, in addition to the choice of the input parameters and the check of the particle spectrum:

i) Renormalization group evolution (RGE) of parameters [17-19], back and forth between the low energy scales, such as $M_{Z}$ and the electroweak symmetry breaking scale, and the high-energy scale, such as the GUT scale or the messenger scale in GMSB models. This RGE is performed for the SM gauge and Yukawa couplings and for the soft SUSY-breaking terms (scalar and gaugino masses, bilinear and trilinear couplings and $\tan \beta$ ) and $\mu$. This procedure has to be iterated in order to include SUSY threshold effects or radiative corrections due to Higgs and SUSY particles.

ii) The consistent implementation of (radiative) electroweak symmetry breaking [20-23] and the calculation of $B$ and $|\mu|$ from the effective scalar potential at one-loop level (plus the leading two-loop contributions). Here, we use the tadpole method to include the loop corrections [22]. The procedure has to be iterated until a convergent value for these two parameters is obtained. [In the first step, the values of $\mu^{2}$ and the electroweak symmetry breaking scale are guessed by using the tree-level potential since no sparticle or Higgs mass has been calculated yet.]

iii) Calculation of the pole masses of the Higgs bosons and the SUSY particles, including the mixing between the current states and the radiative corrections when they are important [24-31]. In this context, we will follow mainly the content and notations of [25], to which we will refer as PBMZ. The latter provides most of the necessary contributions at the oneloop level for the Higgs and sparticle masses, while the leading two-loop corrections to the (neutral) Higgs masses, calculated in Refs. [30-33], are implemented in the form of a separate subroutine twoloophiggs.f (provived by P. Slavich).

The general algorithm is depicted in Figure 1, and we will discuss the various steps in some detail in the following subsections. 


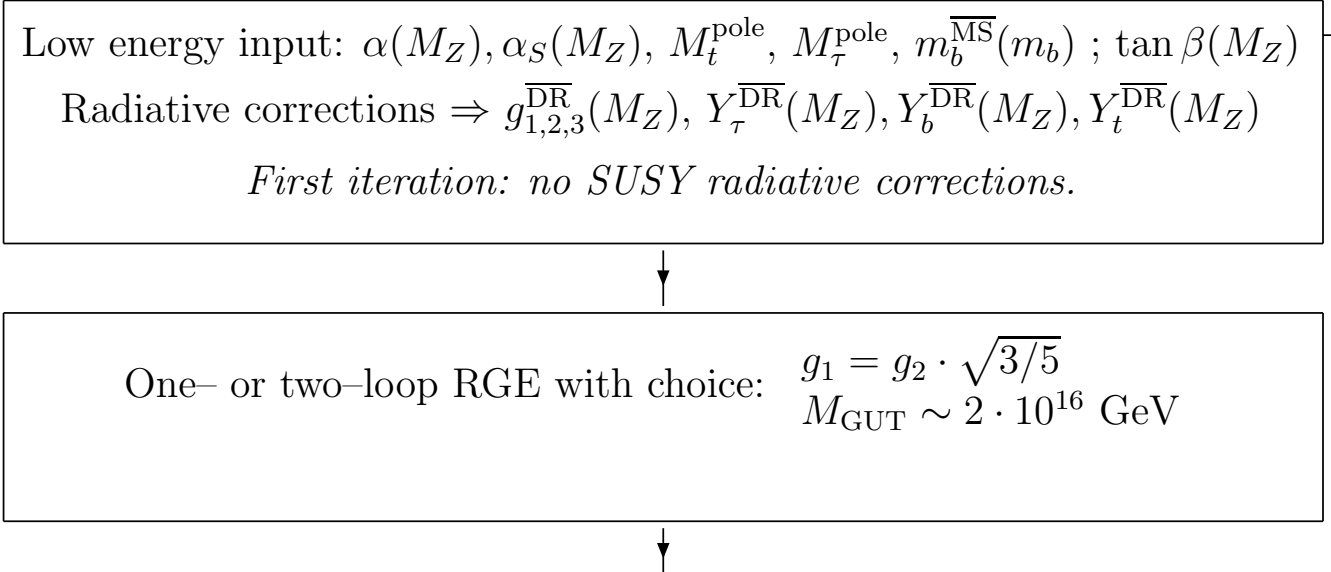

Choice of SUSY-breaking model (mSUGRA, GMSB, AMSB, or pMSSM).

Fix your high-energy input (mSUGRA: $m_{0}, m_{1 / 2}, A_{0}, \operatorname{sign}(\mu)$, etc...).

\section{$\downarrow$}

Run down all parameters with RGE to $m_{Z}$ and $M_{\mathrm{EWSB}}$ scales

First iteration: guess for $M_{\mathrm{EWSB}}$.

$\downarrow$
EWSB: $\mu^{2}, \mu B=F_{\text {non-linear }}\left(m_{H_{u}}, m_{H_{d}}, \tan \beta, V_{\text {loop }}\right)$
$V_{\text {loop }} \equiv$ Effective potential at $1-$ loop with all masses.
First iteration: $V_{\text {loop }}$ not included

Check of consistent EWSB ( $\mu$ convergence, no tachyons, simple CCB/UFB, etc...)

\section{$\downarrow$}

Diagonalization of mass matrices and calculation of masses / couplings

Radiative corrections to the physical Higgs, sfermions, gaugino masses.

First iteration: no radiative corrections.

$\downarrow$

Check of a reasonable spectrum:

- no tachyonic masses (from RGE, EWSB or mix),

-information provided on fine-tuning, $\mathrm{CCB} / \mathrm{UFB}$ conditions,

-calculation of MSSM contributions to: $\Delta \rho,(g-2), b \rightarrow s \gamma$.

Figure 1: Iterative algorithm for the calculation of the SUSY particle spectrum in SuSpect from the choice of input (first step) to the check of the spectrum (last step). The steps are detailed in the various subsections. The EWSB iteration [calculationally fast] on $\mu$ is performed until $\left|\mu_{i}-\mu_{i-1}\right| \leq \epsilon\left|\mu_{i}\right|$ (with $\epsilon \sim 10^{-4}$ ) while the $R G / R C$ "long" iteration [calculationally longer] is performed until some (user specified) accuracy is reached 


\subsection{Calculation of the MSSM parameters at the low scale}

Prior to the calculation of all the relevant terms entering the physical mass spectrum calculation at a low (EWSB) scale, such as typically the soft SUSY-breaking terms in constrained models with boundary conditions at the unification scale, the first important stage is to define a choice of low-energy input parameters and how they are extracted from the present experimental data. This is the purpose of the next subsection.

\subsubsection{Choice and treatment of the SM inputs}

The low-energy (weak) scale boundary conditions set the gauge and Yukawa couplings by matching the running MSSM parameters to the experimental data at a chosen renormalization scale, usually $Q=M_{Z}$. This step is quite involved, requiring to subtract the radiative corrections from the experimental data in order to arrive at the $\overline{\mathrm{DR}}$-renormalized MSSM parameters. Using the formulae of mainly Ref. [25] and references therein, the MSSM $\overline{\mathrm{DR}}$ gauge couplings $g_{1}, g_{2}, g_{3}$ and the electroweak parameter $v$ can be computed at $Q=M_{Z}$ from a set of four experimental input parameters.

The latter can be chosen as: $G_{F}$, the Fermi constant determined from the muon decay; $M_{Z}$, the pole mass of the $Z$ boson; $\alpha_{\mathrm{em}}\left(M_{Z}\right)^{\overline{\mathrm{MS}}}$, the five-flavour SM electromagnetic coupling

at the scale $M_{Z}$ in the $\overline{\mathrm{MS}}$ scheme; $\alpha_{s}\left(M_{Z}\right)^{\overline{\mathrm{MS}}}$, the five-flavour SM strong coupling at the scale $M_{Z}$ in the $\overline{\mathrm{MS}}$ scheme. Then, the running couplings $g_{2}, g_{1}$ are connected to the running $Z$-boson mass $m_{Z}$ by the relation:

$$
m_{Z}^{2}=M_{Z}^{2}+\operatorname{Re} \Pi_{Z Z}^{T}\left(M_{Z}^{2}\right)=\frac{1}{4}\left(g_{1}^{2}+g_{2}^{2}\right) v^{2}
$$

where $\Pi_{Z Z}^{T}\left(M_{Z}^{2}\right)$ is the transverse part of the $Z$ boson self-energy computed at a squared external momentum equal to the squared pole $Z$ boson mass. [Note that according to this input choice, the $W$ boson mass and the parameter $\sin ^{2} \theta_{W}$ are not free parameters but can be derived when needed, e.g. in the $\overline{\mathrm{DR}}$ scheme, from the above defined input parameters using appropriate relations including radiative corrections].

The next MSSM input parameter is $\tan \beta \equiv v_{u} / v_{d}$, the ratio of the vacuum expectation values (VEVs) of the two MSSM neutral Higgs fields, $H_{d}^{0}$ and $H_{u}^{0}$, defined also at the scale $Q=M_{Z}$, with $v_{d}^{2}+v_{u}^{2} \equiv v^{2}$.

Then the Yukawa couplings $Y_{u}(u=u, c, t)$ for the up-type quarks, $Y_{d}(d=d, s, b)$ for the down-type quarks and $Y_{\ell}(\ell=e, \mu, \tau)$ for the leptons are determined in the $\overline{\mathrm{DR}}$ scheme from the corresponding running fermion masses as

$$
Y_{u}=\frac{\sqrt{2} m_{u}}{v \sin \beta}, \quad Y_{d}=\frac{\sqrt{2} m_{d}}{v \cos \beta}, \quad Y_{\ell}=\frac{\sqrt{2} m_{\ell}}{v \cos \beta},
$$

The $\overline{\mathrm{DR}}$ running fermion masses (see e.g. Refs. [62-65]) $m_{f}$ (with $f=u, d, \ell$ ) in eq. (32) can be derived at the one-loop level from the corresponding pole masses $M_{f}$ through the 
relation

$$
m_{f}=M_{f}+\Sigma_{f}\left(M_{f}\right)
$$

where $\Sigma_{f}\left(M_{f}\right)$ is the one-loop fermion self-energy computed at an external momentum equal to the pole mass. In the case of the top quark, the self-energy includes also the leading twoloop standard QCD corrections [63]

$$
m_{t}=M_{t}+\Sigma_{t}\left(M_{t}\right)+\left(\Delta m_{t}\right)^{2-\mathrm{loop}, \mathrm{QCD}}
$$

where the precise expression for $\left(\Delta m_{t}\right)^{2-\text { loop, QCD }}$ depends on the renormalization scheme in which the parameters entering the one-loop self-energy $\Sigma_{t}$ are expressed. Concerning the bottom quark, we follow now the SUSY Les Houches Accord [35] which prescribes to take as input the SM running mass in the $\overline{\mathrm{MS}}$ scheme, $m_{b}\left(m_{b}\right)^{\overline{\mathrm{MS}}}$. In addition, a "resummation" procedure is required (see e.g. Ref. [66]) in order to properly take into account the large QCD corrections, as well as the $\tan \beta$-enhanced SUSY corrections [67], to the relation between the input bottom mass and the corresponding MSSM, $\overline{\mathrm{DR}}$ Yukawa coupling. We extract the latter, via eq. (32), from the MSSM, $\overline{\mathrm{DR}}$ bottom mass $\widehat{m}_{b}$, defined at the scale $Q=M_{Z}$ by the following matching condition:

$$
\widehat{m}_{b} \equiv m_{b}\left(M_{Z}\right)_{\mathrm{MSSM}}^{\overline{\mathrm{DR}}}=\frac{\bar{m}_{b}}{1-\Delta_{b}}
$$

where $\bar{m}_{b} \equiv m_{b}\left(M_{Z}\right)_{\mathrm{SM}}^{\overline{\mathrm{DR}}}$ is the SM, $\overline{\mathrm{DR}}$ bottom mass, obtained by evolving $m_{b}\left(m_{b}\right)^{\overline{\mathrm{MS}}}$ up to the scale $Q=M_{Z}$ with the appropriate RGE, in order to resum the QCD corrections, and then converting it to the $\overline{\mathrm{DR}}$ scheme; $\Delta_{b} \equiv \Sigma_{b}\left(\widehat{m}_{b}\right) / \widehat{m}_{b}$ accounts for the remaining nongluonic corrections, some of which are enhanced by a factor $\tan \beta$. It has been shown [66] that defining the running MSSM bottom mass as in eq. (35) guarantees that the large threshold corrections of $\mathcal{O}\left(\alpha_{s} \tan \beta\right)^{n}$ are included in $\widehat{m}_{b}$ to all orders in the perturbative expansion. Concerning the $\tau$ lepton, the only $\tan \beta$-enhanced corrections to be included are those controlled by the electroweak gauge couplings, from chargino-slepton loops.

Concretely the (present) reference values of the SM input parameters at the weak scale are fixed as follow. We take for the electroweak and strong parameters [41]:

$$
\begin{gathered}
G_{F}=1.1663910^{-5} \mathrm{GeV}^{-2}, \quad M_{Z}=91.1876 \mathrm{GeV}, \\
\alpha_{\mathrm{em}}^{-1}\left(M_{Z}\right)^{\overline{\mathrm{MS}}}=127.934, \quad \alpha_{s}\left(M_{Z}\right)^{\overline{\mathrm{MS}}}=0.1172
\end{gathered}
$$

and for the third-generation fermion masses the values [41,68]:

$$
M_{t}=178.0 \mathrm{GeV}, \quad m_{b}\left(m_{b}\right)^{\overline{\mathrm{MS}}}=4.25 \mathrm{GeV}, \quad M_{\tau}=1.777 \mathrm{GeV} .
$$

Of course, for maximal flexibility, all those SM input default values in eqs. (36/37) can be changed at will in the input file. 
Once the Supersymmetric particle spectrum has been obtained [see below], we include all the important SUSY radiative corrections to the gauge and Yukawa couplings, via their above defining relations from input parameters, e.g. eqs. (3134) or (35), where the SUSY particle masses enter the expression of e.g. $\Pi_{Z Z}^{T}$ in eq. (31) and $\Sigma_{t}$ in eq. (34). This necessarily implies an iterative procedure, since the values of the SUSY particle spectrum depend, among other thing, on the precise values of the gauge and Yukawa couplings via the RGE typically. The iteration is to be performed until a sufficiently stable final SUSY spectrum is obtained [and where the very first iteration is done with only SM radiative corrections included in eqs. (3134), before SUSY particle masses are defined, see the general algorithm overview in Fig. 1]. In the case of the Yukawa couplings, we include all relevant SUSY corrections to the third generation fermion masses. For the bottom quark ( $\tau$ lepton) mass, we include the SUSY-QCD and stop-chargino (sneutrino-chargino) one-loop corrections at zero-momentum transfer [66] which, according to PBMZ, is an extremely good approximation. These corrections to the $b$ and $\tau$ masses are enhanced by terms $\propto \mu \tan \beta$ and can be rather large. For the top quark, the inclusion of only the leading corrections at zero momentum transfer is not an accurate approximation, and we include the full one-loop SUSY-QCD [i.e stop and gluino loops] and electroweak [i.e. with gauge, Higgs boson and chargino/neutralino exchange] corrections à la PBMZ [25].

\subsubsection{Renormalization Group Evolution}

All gauge and (third generation) Yukawa couplings are then evolved up to the GUT scale using the two-loop MSSM RGEs $[19,20]$ in the $\overline{\mathrm{DR}}$ scheme, with the contribution of all the MSSM particles in the relevant beta-functions. The GUT scale, $M_{\mathrm{GUT}} \simeq 2 \cdot 10^{16} \mathrm{GeV}$ can be either fixed by hand or, by appropriate user's choice in the input file, calculated consistently to be the scale at which the electroweak gauge coupling constants [with the adequate normalization] unify, $g_{1}=g_{2} \cdot \sqrt{3 / 5}$. In contrast, we do not enforce exact $g_{2}=g_{3}$ unification at the GUT scale and assume that the small discrepancy, of at most a few percent, is accounted for by unknown GUT-scale threshold corrections [69].

One can then chose the parameter $\tan \beta$, given at the scale $M_{Z}$, the sign of the $\mu$ parameter and, depending on the chosen model, the high energy and the low energy input. For instance, one can set the high-energy scale $E_{\mathrm{High}}$, which in mSUGRA or AMSB can be either forced to be $M_{\mathrm{GUT}}$ [the scale at which $g_{1}$ and $g_{2}$ unify] or chosen at will [any particular intermediate scale between $M_{Z}$ and $M_{\mathrm{GUT}}$ can be allowed in general and in the case of the GMSB model this scale corresponds to the messenger scale $M_{\text {mes }}$ ]. Similarly the low energy scale $E_{\text {Low }}$, where the RGEs start or end may be chosen [it is in general taken to be $M_{Z}$ or the EWSB scale to be discussed later]. The additional input in the various models are:

- $\underline{\text { mSUGRA: }}$ the universal trilinear coupling $A_{0}$, the common scalar mass $m_{0}$ and the common gaugino mass $m_{1 / 2}$, all defined at the scale $M_{\mathrm{GUT}}$. 
- AMSB: the common scalar mass $m_{0}$, the gravitino mass $m_{3 / 2}$ and the set of coefficients $c_{S_{i}}$ for the non-anomalous contributions, to be as general as possible.

- GMSB: the scale $\Lambda$, the messenger scale $M_{\text {mes }}$ which corresponds to $E_{\mathrm{High}}$, as well as the numbers of messengers $n_{q}$ and $n_{l}$.

- pMSSM with boundary conditions: the various soft SUSY-breaking parameters listed in section 2.2 [21 parameters in total, in addition to $\tan \beta]$ defined at the scale $E_{\text {High }}$. These input can also be chosen at will at the low-energy scale $E_{\text {Low }}$ which has also to be provided as a necessary input. For this pMSSM input choice we stress that all the (scale-dependent) soft SUSY-breaking parameters are implicitly understood to be defined at the given arbitrary low scale $E_{\mathrm{Low}}$. In particular, the code will perform RGE consistently between the scale $Q=M_{Z}$ and $Q=E_{\text {Low }}$, to match e.g. with the SM input defined at $Q=m_{Z}$, as well as the RGE between $Q=E_{\text {Low }}$ and the EWSB scale $M_{\mathrm{EWSB}}$, while the RGE from a high scale $E_{\mathrm{High}}$ down to $M_{\mathrm{EWSB}}$ are not relevant in this case and are thus switched off. Note also that here, a very convenient option is provided which allows to trade the input parameters $M_{H_{u}}^{2}$ and $M_{H_{d}}^{2}$ with the more "physical" parameters $M_{A}$ (pole mass) and $\mu\left(E_{\text {low }}\right)$ [again in such a way that EWSB is consistently realized, with a warning flag whenever it is not the case].

Given these boundary conditions, all the soft SUSY breaking parameters and couplings are evolved down to the EWSB and $M_{Z}$ scales, using either the one- or (preferably) full two-loop RGE options ${ }^{2}$. Our default choice for the EWSB scale is the geometric mean of the two top squark running masses (in the $\overline{\mathrm{DR}}$ scheme),

$$
M_{\mathrm{EWSB}}=\left(m_{\tilde{t}_{1}} m_{\tilde{t}_{2}}\right)^{1 / 2}
$$

which minimizes the scale dependence of the one-loop effective potential [23] discussed below [at first iteration where the stop masses have not yet been calculated, we use the geometric mean of the soft SUSY-breaking stop masses instead as a first guess]. Note, however, that any other arbitrary values of the EWSB scale can be chosen easily by an appropriate input setting. Since $\tan \beta$ is defined at $M_{Z}$, the vevs have to be evolved down from $M_{\mathrm{EWSB}}$ to $M_{Z}$.

Once the SUSY spectrum is calculated [see below], the heavy (s)particles are taken to contribute to reevaluate the gauge and Yukawa couplings at the scale $Q=M_{Z}$, as discussed in the previous subsection, and the necessary iterative procedure thus also includes the RGEs.

\footnotetext{
${ }^{2}$ The full two-loop RGEs in the MSSM [70], have been implemented for the slepton and quark masses in the latest version of SuSpect. In the previous versions, the two-loop RGEs were included for all terms except for the latter soft SUSY-breaking masses. These higher order effects can have a non-negligible impact [95] in some regions of the MSSM parameter space.
} 


\subsubsection{Electroweak Symmetry Breaking}

At some stage, we require that the electroweak symmetry is broken radiatively and use eq. (111) to determine the parameters $\mu^{2}\left(M_{\mathrm{EWSB}}\right)$ and $B\left(M_{\mathrm{EWSB}}\right)$. It is well known that the one-loop radiative corrections to the Higgs potential play a major role in determining the values of these two parameters, which at tree level are given in terms of the soft SUSYbreaking masses of the two Higgs doublet fields. We treat these corrections using the tadpole method. This means that we can still use eq. (11) to determine $\mu^{2}\left(M_{\text {EWSB }}\right)$, one simply has to add one-loop tadpole corrections $t_{u}, t_{d}[22,25]$

$$
m_{H_{u}}^{2} \rightarrow m_{H_{u}}^{2}-t_{u} / v_{u} \text { and } m_{H_{d}}^{2} \rightarrow m_{H_{d}}^{2}-t_{d} / v_{d}
$$

We include the dominant third generation fermion/sfermion loops, as well as sub-dominant contributions from sfermions of the first two generations, gauge bosons, the MSSM Higgs bosons, charginos and neutralinos ${ }^{3}$, with the running parameters evaluated at $M_{\mathrm{EWSB}}$. Note that we also include the leading two-loop tadpole corrections, as calculated in Refs. [30-32], to be consistent in particular with the subsequent calculation of the physical neutral Higgs masses including the leading two-loop contributions.

As far as the determination of $\mu^{2}$ and $B \mu$ is concerned, this is equivalent (at the considered loop level) to computing the full effective potential at scale $M_{\mathrm{EWSB}}$. Since $|\mu|$ and $B$ affect the masses of some (s)particles appearing in these corrections, this gives a non-linear equation for $|\mu|$ (see Fig. 1), which is solved by a standard iteration algorithm until stability is reached and a consistent value of $\mu$ is obtained. From a practical point of view, this requires only three or four iterations for an accuracy of $\mathcal{O}\left(10^{-4}\right)$, if one starts from the values of $|\mu|$ and $B$ as determined from minimization of the $\mathrm{RG}$-improved tree-level potential at scale $M_{\mathrm{EwSB}}$ and the procedure is extremely fast in CPU as compared to the (iterated) RGE calculation.

At this stage, SuSpect includes a check on whether the complete scalar potential has charge and/or color breaking (CCB) minima which can be lower than the electroweak minimum, or whether the tree-level scalar potential is unbounded from below (UFB). In the present version of the code, we consider only the following simple (tree-level) criteria [40]

$$
\begin{array}{ll}
\text { CCB1 : } & A_{f}^{2}<3\left(m_{\tilde{f}_{L}}^{2}+m_{\tilde{f}_{R}}^{2}+\mu^{2}+m_{H_{u}}^{2}\right) . \\
\mathrm{UFB} 1: & m_{H_{u}}^{2}+m_{H_{d}}^{2} \geq 2|B \mu| \text { at scale } Q^{2}>M_{\mathrm{EWSB}}^{2}
\end{array}
$$

where $f$ denotes any of the three fermion generations. Eq. (40) ensures that there is no deep CCB breaking minimum [due to very small Yukawa couplings] in some D-flat directions. One can either take this as a consistency necessary constraint on the MSSM parameters, or disregard it appealing to the fact that such minima are usually well separated from the electroweak minimum so that the latter can be reasonably stable at cosmological scales ${ }^{4}$.

\footnotetext{
${ }^{3}$ The contributions of the charginos and neutralinos can be rather sizable and are very important to minimize the scale dependence of the one-loop effective potential [24].

${ }^{4}$ But one would still lack for a compelling reason why the EW minimum is chosen in the first place.
} 
For the third generation and in particular in the top sector, the CCB minimum is not much deeper than the electroweak minimum, since $Y_{t}$ is not very small, and not much separated from it. In this case one should apply eq. (40) with some caution since tunneling effects can be important. On the other hand the "boundedness-from-below" condition of eq. (41) is actually an indication of possible dangerous non physical minima which could form when radiative corrections are included. At any rate, since both eqs. (40) and (41) are merely treelevel conditions, they should be checked at the highest energy scale. Note that all subsequent calculations (i.e. the sparticle and Higgs masses) are still performed, even if these conditions are not fulfilled, but a warning flag is given in the output file. An upcoming version of SuSpect will have more sophisticated treatments, taking into account loop corrections [71] as well as the geometric configurations of the true minima [72,73] as will be discussed later.

Finally, SuSpect provides appropriate (rejection) flags for any input choice in the parameter space which lead to tachyonic pseudo-scalar Higgs boson or sfermion masses:

$$
\text { No Tachyon : } \quad M_{A}^{2}>0, m_{\tilde{f}}^{2}>0 .
$$

The electroweak symmetry breaking mechanism is assumed to be consistent when all these conditions are satisfied. Note however that for some input parameter choice such problems may occur temporarily i.e. before a sufficiently stable SUSY spectrum is obtained, and accordingly the SuSpect algorithm allows the calculation to be nevertheless performed until the very last iteration before eventually issuing a rejection flag.

\subsection{Calculation of the physical particle masses}

Once all the soft SUSY-breaking terms are obtained and eventually EWSB is radiatively realized [as should be the case in unified models] one can then calculate all the physical particle masses. As already mentioned the whole procedure (namely, RGE + EWSB + spectrum calculation) is iterated a number of time until stability is reached (see the overall algorithm in Fig 1), in order to take into account realistic and stable particle masses in the "threshold" corrections using the expressions given in Ref. [25], to the gauge and Yukawa couplings at the scale $Q=M_{Z}$.

Our conventions for the mass matrices in the gaugino, sfermion and Higgs sectors will be specified below. We basically follow the conventions of PBMZ with some important exceptions: $(i)$ The $\mu$ parameter is defined with the opposite sign (see below). ( $i i$ ) The vevs are different by a factor $\sqrt{2}$ and in our case $v=174.1 \mathrm{GeV}$. (iii) The sfermion masses are defined such that $\tilde{f}_{1}$ and $\tilde{f}_{2}$ are, respectively, the lightest and the heaviest one. (iv) The matrices diagonalizing the chargino and neutralino mass matrices are taken to be real.

For the calculation of the physical masses and the implementation of the radiative corrections, the various sectors of the MSSM are then treated as follows [with some details on the notation and conventions we use]: 


\subsubsection{The sfermion sector}

In the third generation sfermion sector $[\tilde{t}, \tilde{b}, \tilde{\tau}]$, mixing between "left" and "right" current eigenstates is included [74]. The radiatively corrected running $\overline{\mathrm{DR}}$ fermion masses [essentially the Yukawa coupling times vevs] at scale $M_{\text {EWsB }}$ are employed in the sfermion mass matrices [this is important at large $\tan \beta$, where these corrections can be quite sizable]. As mentioned above, contrary to PBMZ, the masses are defined such that $m_{\tilde{f}_{1}}$ and $m_{\tilde{f}_{2}}$ correspond to the mass of respectively, the lightest and the heaviest sfermion and therefore care should be made in interpreting the sfermion mixing angle $\theta_{\tilde{f}}$ as compared to PBMZ. [Note that a protection which prevents negative mass squared for third generation sfermions in the presence of too large mixing is provided.] The sfermion mass matrices are given by:

$$
M_{\tilde{f}}^{2}=\left[\begin{array}{cc}
m_{\tilde{f}_{L}}^{2}+\left(I_{f}^{3}-e_{f} s_{W}^{2}\right) M_{Z}^{2} \cos 2 \beta+m_{f}^{2} & m_{f}\left(A_{f}-\mu r_{f}\right) \\
m_{f}\left(A_{f}-\mu r_{f}\right) & m_{\tilde{f}_{R}}^{2}-e_{f} s_{W}^{2} M_{Z}^{2} \cos 2 \beta+m_{f}^{2}
\end{array}\right]
$$

where $m_{\tilde{f}_{L, R}}, A_{f}, \mu$ and $m_{f}$ are respectively, the $\overline{\mathrm{DR}}$ soft SUSY scalar masses, trilinear couplings, higgsino mass parameter and running fermion masses at the scale $M_{\text {EwsB }}$ and $r_{b}=r_{\tau}=1 / r_{t}=\tan \beta$. These matrices are diagonalized by orthogonal matrices; the mixing angles $\theta_{f}$ and the squark eigenstate masses are determined uniquely, at the relevant energy scales $^{5}$ by

$$
\tan 2 \theta_{f}=\frac{2 m_{f}\left(A_{f}-\mu r_{f}\right)}{m_{\tilde{f}_{L}}^{2}-m_{\tilde{f}_{R}}^{2}+I_{f}^{3} M_{Z}^{2} \cos 2 \beta}
$$

or

$$
\tan \theta_{f}=\frac{m_{\tilde{f}_{1}}^{2}-m_{\tilde{f}_{2}}^{2}+m_{\tilde{f}_{R}}^{2}-m_{\tilde{f}_{L}}^{2}-I_{f}^{3} M_{Z}^{2} \cos 2 \beta}{2 m_{f}\left(A_{f}-\mu r_{f}\right)}
$$

and

$$
m_{\tilde{f}_{1,2}}^{2}=m_{f}^{2}+\frac{1}{2}\left[m_{\tilde{f}_{L}}^{2}+m_{\tilde{f}_{R}}^{2} \mp \sqrt{\left(m_{\tilde{f}_{L}}^{2}-m_{\tilde{f}_{R}}^{2}+I_{f}^{3} M_{Z}^{2} \cos 2 \beta\right)^{2}+4 m_{f}^{2}\left(A_{f}-\mu r_{f}\right)^{2}}\right]
$$

Our convention for the mixing angles is

$$
\left(\begin{array}{c}
\tilde{f}_{1} \\
\tilde{f}_{2}
\end{array}\right)=\left[\begin{array}{cc}
\cos \theta_{f} & \sin \theta_{f} \\
-\sin \theta_{f} & \cos \theta_{f}
\end{array}\right]\left(\begin{array}{c}
\tilde{f}_{L} \\
\tilde{f}_{R}
\end{array}\right)
$$

where $\tilde{f}_{i}$ denote the mass eigenstates, and $\tilde{f}_{L, R}$ the chiral states. Equations $(45,46)$ ensure simultaneously that each $m_{\tilde{f}_{i}}$ given in (44) is indeed the mass corresponding to the state $\tilde{f}_{i}$ and that $\tilde{f}_{1}$ is always the lightest state. In practice, the possible ambiguity, when $\theta_{f}$ is

\footnotetext{
${ }^{5}$ Note that the sfermion masses and mixing angles are also calculated for consistency by SuSpect at the $M_{Z}$ scale as well since they enter the various radiative corrections to the gauge and Yukawa couplings.
} 
determined from $\frac{1}{2} \arctan \left(\tan 2 \theta_{f}\right)$, is lifted in SuSpect by requiring that $\tan \theta_{f}$ has always an opposite sign to $A_{f}-\mu r_{f}$, as can be seen from eq. $(45)^{6}$.

The radiative corrections to the sfermion masses are included according to Ref. [25], i.e. only the QCD corrections for the superpartners of light quarks [including the bottom squark] plus the leading electroweak corrections to the two top squarks; the small electroweak radiative corrections to the slepton masses [which according to PBMZ are at the level of one percent] have been neglected in the present version.

\subsubsection{The gaugino sector}

The $2 \times 2$ chargino and $4 \times 4$ neutralino mass matrices depend on the $\overline{\mathrm{DR}}$ parameters $M_{1}, M_{2}, \mu$ at the scale $M_{\mathrm{EWSB}}$ and on $\tan \beta$. The chargino mass matrix given by:

$$
\mathcal{M}_{C}=\left[\begin{array}{cc}
M_{2} & \sqrt{2} M_{W} \sin \beta \\
\sqrt{2} M_{W} \cos \beta & \mu
\end{array}\right]
$$

is diagonalized by two real matrices $U$ and $V$. The chargino masses are obtained analytically, with the convention that $\chi_{1}^{+}$is the lightest state.

The neutralino mass matrix, in the $\left(-i \tilde{B},-i \tilde{W}_{3}, \tilde{H}_{1}^{0}, \tilde{H}_{2}^{0}\right)$ basis, has the form

$$
\mathcal{M}_{N}=\left[\begin{array}{cccc}
M_{1} & 0 & -M_{Z} s_{W} \cos \beta & M_{Z} s_{W} \sin \beta \\
0 & M_{2} & M_{Z} c_{W} \cos \beta & -M_{Z} c_{W} \sin \beta \\
-M_{Z} s_{W} \cos \beta & M_{Z} c_{W} \cos \beta & 0 & -\mu \\
M_{Z} s_{W} \sin \beta & -M_{Z} c_{W} \sin \beta & -\mu & 0
\end{array}\right]
$$

It is diagonalized using analytical formulae [75] by a single matrix $Z$ which is chosen to be real, leading to the fact that some (in general one) of the neutralino eigenvalues is negative. The physical masses are then the absolute values of these eigenvalues with some reordering such that the neutralinos $\chi_{1,2,3,4}^{0}$ are heavier with increasing subscript and $\chi_{1}^{0}$ is the lightest neutralino.

For the gluino, the running $\overline{\mathrm{DR}}$ mass $m_{\tilde{g}}$ at scale $M_{\mathrm{EWSB}}$ is identified with $M_{3}\left(M_{\mathrm{EWSB}}\right)$

$$
m_{\tilde{g}}^{\text {tree }}=M_{3}\left(M_{\mathrm{EWSB}}\right)
$$

The full one-loop QCD radiative corrections to the gluino mass are incorporated [20], while in the charginos/neutralinos case the radiative corrections to the masses are simply included in the gaugino and higgsino limits, which is a very good approximation [25].

\footnotetext{
${ }^{6}$ In other parts of the code where sfermion masses and mixing angles are used internally, for instance when entering in loop corrections, only the first equation in (44) is used to determine the mixing angles. In this case the consistency is guaranteed through the calculation of the eigenmasses directly from the mixing angles in the form $\left(M_{\tilde{f}}^{2}\right)_{11} \cos ^{2} \theta_{f}+\left(M_{\tilde{f}}^{2}\right)_{22} \sin ^{2} \theta_{f} \pm\left(M_{\tilde{f}}^{2}\right)_{12} \sin 2 \theta_{f}$, rather than from (45). Of course, the convention $m_{\tilde{f}_{1}}^{2}<m_{\tilde{f}_{2}}^{2}$ is not necessarily valid here.
} 


\subsubsection{The Higgs sector}

In the latest version of SuSpect we have performed an up-to-date and more precise determination of the physical Higgs masses including, in particular, the leading two-loop contributions [30-33] to the neutral Higgs masses and tadpoles. The running $\overline{\mathrm{DR}}$ mass of the pseudoscalar Higgs boson at the scale $M_{\mathrm{EWSB}}, \bar{M}_{A}$, is obtained from the soft SUSY-breaking Higgs mass terms evolved from RGE at the scale $M_{\text {EWSB }}$ and including the loop tadpole corrections $[25]$

$$
\bar{M}_{A}^{2}\left(M_{\mathrm{EWSB}}\right)=\frac{1}{\cos 2 \beta}\left(m_{H_{d}}^{2}-\frac{t_{d}}{v_{d}}-m_{H_{u}}^{2}+\frac{t_{u}}{v_{u}}\right)-\bar{M}_{Z}^{2}+\sin ^{2} \beta \frac{t_{d}}{v_{d}}+\cos ^{2} \beta \frac{t_{u}}{v_{u}}
$$

This mass, together with the $Z$ boson mass $\bar{M}_{Z}$ at scale $M_{\text {EwsB }}$, are then used as inputs in the $\mathrm{CP}$-even Higgs boson $2 \times 2$ mass matrix $\mathcal{M}_{S}$. Including the dominant contributions of the self-energies of the unrotated $\mathrm{CP}$-even neutral Higgs fields $H_{u}^{0}$ and $H_{d}^{0}$ (as well as the tadpole contributions), this matrix reads at a given scale $q^{2}$

$$
\mathcal{M}^{S}\left(q^{2}\right)=\left[\begin{array}{cc}
\bar{M}_{Z}^{2} \cos \beta^{2}+\bar{M}_{A}^{2} \sin ^{2} \beta-s_{11}\left(q^{2}\right) & -\frac{1}{2}\left(\bar{M}_{Z}^{2}+\bar{M}_{A}^{2}\right) \sin 2 \beta-s_{12}\left(q^{2}\right) \\
-\frac{1}{2}\left(\bar{M}_{Z}^{2}+\bar{M}_{A}^{2}\right) \sin 2 \beta-s_{12}\left(q^{2}\right) & \bar{M}_{Z}^{2} \sin ^{2} \beta+\bar{M}_{A}^{2} \cos ^{2} \beta-s_{22}\left(q^{2}\right)
\end{array}\right]
$$

One obtains the running $\mathrm{CP}$-even Higgs boson masses in terms of the matrix elements $\mathcal{M}_{i j}^{S}$

$$
\bar{M}_{h, H}^{2}=\frac{1}{2}\left[\mathcal{M}_{11}^{S}+\mathcal{M}_{22}^{S} \mp \sqrt{\left(\mathcal{M}_{11}^{S}-\mathcal{M}_{22}^{S}\right)^{2}+4\left(\mathcal{M}_{12}^{S}\right)^{2}}\right]
$$

The mixing angle $\alpha$ which diagonalizes the matrix $\mathcal{M}^{S}$ and rotates the fields $H_{u}^{0}, H_{d}^{0}$ into the physical $\mathrm{CP}$-even Higgs boson fields $h, H$

$$
\left(\begin{array}{c}
H \\
h
\end{array}\right)=\left(\begin{array}{cc}
\cos \alpha & \sin \alpha \\
-\sin \alpha & \cos \alpha
\end{array}\right)\left(\begin{array}{c}
H_{d}^{0} \\
H_{u}^{0}
\end{array}\right)
$$

is given by

$$
\sin 2 \alpha=\frac{2 \mathcal{M}_{12}^{S}}{\bar{M}_{H}^{2}-\bar{M}_{h}^{2}} \quad, \cos 2 \alpha=\frac{\mathcal{M}_{11}^{S}-\mathcal{M}_{22}^{S}}{\bar{M}_{H}^{2}-\bar{M}_{h}^{2}} \quad\left(-\frac{\pi}{2}<\alpha<\frac{\pi}{2}\right)
$$

The running charged Higgs boson mass at the EWSB scale is given by

$$
\bar{M}_{H^{ \pm}}^{2}=\bar{M}_{A}^{2}+\bar{M}_{W}^{2}-\sin ^{2} \beta \frac{t_{d}}{v_{d}}-\cos ^{2} \beta \frac{t_{u}}{v_{u}}
$$

The pole masses of all the Higgs bosons are then obtained by including the self-energy corrections evaluated at the masses of the Higgs bosons themselves.

In the evaluation of the radiative corrections in the MSSM Higgs sector which are known to be very important [27], we have made several options available:

(i) Approximate one-loop and two-loop contributions to the self-energies (and tadpole) corrections $s_{i j}$ in the mass matrix $\mathcal{M}^{S}$. These expressions, given in Ref. [29], provide a rather 
good approximation (at the few percent level) for the masses $M_{h}$ and $M_{H}$ and the angle $\alpha$ at least for a reasonable range and not too extreme values of input parameters. Since it makes the program running faster, this approximation may be convenient in cases where a very precise determination of the Higgs masses may not be mandatory.

(ii) A full one-loop calculation of the Higgs masses, following essentially the expressions of Ref. [25]. In this case, the tadpoles are consistently evaluated at the one-loop level only.

(iii) A full one-loop plus leading two-loop calculation controlled by the third generation Yukawa couplings and the strong gauge coupling, derived in Refs. [30-33]. Note that the calculation is entirely performed in the $\overline{\mathrm{DR}}$ scheme, therefore different from the on-shell scheme calculation performed in FeynHiggsFast of Heinemeyer, Hollik and Weiglein [28,29]. The comparison of results from (ii) and (iii) can be useful in order to see the effects from two-loop contributions. For a recent detailed analysis of the Higgs masses in various models and a comparison between SuSpect and other codes, we refer to Ref. [34].

\subsection{Theoretical and experimental constraints on the spectra}

Once the SUSY and Higgs spectrum is calculated, one can check that some theoretical and experimental requirements are fulfilled. Examples of theory requirements are for instance, the absence of charge and color breaking (CCB) minima and that the potential is not unbounded from below (UFB), the absence of too much fine-tuning (FT) in the determination of the masses of the $Z$ boson from EWSB as well as in the determination of the top quark mass. For experimental requirements on the spectrum, one can demand for instance that it does not lead to large radiative corrections to the precisely measured electroweak parameters or too large values for the anomalous magnetic moment of the muon and the branching ratio of the radiative decay of the $b$-quark. SuSpect provides such tests in the form of warning or error flags in the output file.

\subsubsection{CCB and UFB}

As explained previously, in Suspect, the EWSB conditions are consistently implemented by iteration on the parameters $\mu$ and $B$ and the occurrence of a local minimum is checked numerically. In the same time one needs to check for the non existence of deep CCB minima or UFB directions. Avoiding such cases may put strong constraints on the model and we mentioned in section 3.2.3 that we have already implemented two simple CCB and UFB conditions [40] and the program gives a warning flag when they are not satisfied.

In a next version of the code, to be released in a near future, we will address the question of CCB minima and UFB directions in the most complete possible way, given the present state of the art. Three complementary features should be considered in relation to the CCB minima: ( $i$ ) the directions in the space of scalar fields along which such minima can develop (ii) whether they are lower than the EWSB minimum (iii) whether the EWSB (then 
false) vacuum can still be sufficiently stable. In Ref. [71] a systematic study of point $(i)$ has been carried out considering subspaces involving the fields $H_{u}, \tilde{Q}_{u}, \tilde{u}_{R}\left(H_{d}\right.$ and possibly $\tilde{L})$. However, the identified D-flat directions contain the true minima only in the case of universal scalar soft masses at the low energy relevant scales, otherwise they constitute only sufficient conditions for the occurrence of CCB minima. While such directions provide very good approximations for the first two generations, special attention should be paid to the third generation sector as was stressed in Ref. [72]. This is relevant in particular to codes like SuSpect where various SUSY model assumptions can be considered, including nonuniversality. Furthermore, the check of point (ii) as done in Ref. [71] involves a numerical scan over field values. Actually there are cases where field-independent conditions can be obtained even in the case of 5 -field directions $H_{u}, \tilde{Q}, \tilde{u}_{R}, H_{d}, \tilde{L}$, leading to faster algorithms; see Ref. [72] and unpublished study. We will thus optimize in SuSpect the various available complementary approaches. Point (iii) has also its importance as it can increase the phenomenologically allowed regions of the MSSM parameter space. Some simple criteria will be encoded, following for instance Ref. [73]. Finally, the UFB directions as identified in Ref. [71], in particular UFB-3, lead to very strong constraints. Nonetheless, there is still room for some improvements by optimizing the criterion of "deepest direction", leading in some cases to even stronger constraints which will be also implemented in SuSpect.

\subsubsection{Fine-tuning}

One of the main motivations for low energy SUSY is that it solves technically the hierarchy and naturalness problems. However, since the $Z$ boson mass is determined by the soft SUSYbreaking masses $M_{H_{u}}^{2}, M_{H_{u}}^{2}$ and the parameter $\mu^{2}$ as can be seen from eq. (11), naturalness requires that there are no large cancellations when these parameters are expressed in terms of the fundamental parameters of the model [for instance $m_{0}, m_{1 / 2}, \mu, B$ in mSUGRA], otherwise fine-tuning is re-introduced $[19,77,78]$. A similar problem occurs in the case of the top quark mass, since it is related to the top Yukawa coupling and $\tan \beta$. Various criteria for quantifying the degree of fine-tuning in the determination of $M_{Z}$ and $m_{t}$ have been proposed and some subjectivity is involved in the statement of how much fine tuning can be allowed. Therefore, in our case, we simply evaluate the sensitivity coefficients for $M_{Z}^{2}$ and $m_{t}$ with respect to a given parameter $a[78]$

$$
\begin{aligned}
\frac{\delta M_{Z}^{2}}{M_{Z}^{2}} & =C\left(M_{Z}^{2}, a\right) \frac{\delta a}{a} \\
\frac{\delta m_{t}}{m_{t}} & =C\left(m_{t}, a\right) \frac{\delta a}{a}
\end{aligned}
$$

and leave to the user the decision of whether the amount of fine-tuning [large values of the $C$ coefficients] is bearable or not. We evaluate only the fine-tuning with respect to variations 
of the parameters $\mu^{2}$ and $B \mu$, for which the coefficients take the simple form:

$$
\begin{aligned}
& \text { FT1MZ : } C\left(M_{Z}^{2}, \mu^{2}\right)=\frac{2 \mu^{2}}{M_{Z}^{2}}\left[1+t_{\beta} \frac{4 \tan ^{2} \beta\left(\bar{m}_{1}^{2}-\bar{m}_{2}^{2}\right)}{\left(\bar{m}_{1}^{2}-\bar{m}_{2}^{2}\right) t_{\beta}-M_{Z}^{2}}\right] \\
& \text { FT1MZ : } C\left(M_{Z}^{2}, B \mu\right)=4 t_{\beta} \tan ^{2} \beta \frac{\bar{m}_{1}^{2}-\bar{m}_{2}^{2}}{M_{Z}^{2}\left(\tan ^{2} \beta-1\right)^{2}} \\
& \text { FT1MT : } \quad C\left(m_{t}, \mu^{2}\right)=\frac{1}{2} C\left(M_{Z}^{2}, \mu^{2}\right)+\frac{2 \mu^{2}}{\bar{m}_{1}^{2}+\bar{m}_{2}^{2}} \frac{1}{\tan ^{2} \beta-1} \\
& \text { FT1MT : } \quad C\left(m_{t}, B \mu\right)=\frac{1}{2} C\left(M_{Z}^{2}, B \mu\right)+\frac{1}{1-\tan ^{2} \beta}
\end{aligned}
$$

with $t_{\beta}=\left(\tan ^{2} \beta+1\right) /\left(\tan ^{2} \beta-1\right)$. Further fine-tuning tests can be made, in particular with respect to the $t, b$ Yukawa couplings and are planned to be included in future versions.

\subsubsection{Electroweak precision measurements}

Loops involving Higgs and SUSY particles can contribute to electroweak observables which have been precisely measured at LEP, SLC and the Tevatron. In particular, the radiative corrections to the self-energies of the $W$ and $Z$ bosons, $\Pi_{W W}$ and $\Pi_{Z Z}$, might be sizable if there is a large mass splitting between some particles belonging to the same $\mathrm{SU}(2)$ doublet; this can generate a contribution which grows as the mass squared of the heaviest particle. The dominant contributions to the electroweak observables, in particular the $W$ boson mass and the effective mixing angle $s_{W}^{2}$, enter via a deviation from unity of the $\rho$ parameter [79], which measures the relative strength of the neutral to charged current processes at zero momentum transfer, i.e. the breaking of the global custodial SU(2) symmetry:

$$
\rho=(1-\Delta \rho)^{-1} ; \Delta \rho=\Pi_{Z Z}(0) / M_{Z}^{2}-\Pi_{W W}(0) / M_{W}^{2}
$$

Most of the MSSM contributions to the $\rho$ parameter are small, $\Delta \rho \lesssim 10^{-4}$ [80]. In the case of the Higgs bosons, the contributions are logarithmic, $\sim \alpha \log \left(M_{h} / M_{Z}\right)$, and are similar to those of the SM Higgs boson [and identical in the decoupling limit]. The chargino and neutralino contributions are small because the only terms in the mass matrices which could break the custodial $\mathrm{SU}(2)$ symmetry are proportional to $M_{W}$. Since in general, first/second generation sfermions are almost degenerate in mass, they also give very small contributions to $\Delta \rho$. Therefore, only the third generation sfermion sector can generate sizable corrections to the $\rho$ parameter, because of left-right mixing and [in case of the stop] the SUSY contribution $\propto m_{f}^{2}$ leads to a potentially large splitting between the sfermion masses.

We have thus calculated $\Delta \rho$ in the MSSM, taking into account only the contributions of the third generation sfermions. We include full mixing and in the case of the stop/sbottom doublet, also the two-loop QCD corrections due to gluon exchange and the correction due to gluino exchange in the heavy gluino limit, which can increase the contribution by $30 \%$ or so [81]. One may then require the SUSY contribution not to exceed e.g. two standard 
deviations from the SM expectation [82]: $\Delta \rho$ (SUSY) $\lesssim 2 \cdot 10^{-3}$. The precise value of $\Delta \rho$ as calculated by SuSpect for any choice of parameters is written in the output files.

\subsubsection{The muon (g-2)}

The muon $(g-2)$ anomalous magnetic moment has been very precisely measured and thus generally can set strong constraints on the additional contribution from SUSY particles. The latest experimental value is [83]:

$$
\left(g_{\mu}-2\right) \equiv a_{\mu}^{\exp }=(11659208 \pm 6) 10^{-10},
$$

The theoretical value predicted in the SM, including the QED, QCD and electroweak corrections, has some uncertainties from the determination of the hadronic vacuum polarization contributions. In particular, at the time of this writing, the SM theoretical prediction depends quite crucially on whether one takes into account the combined measurement from $e^{+} e^{-}$and $\tau$ decays data, or from $e^{+} e^{-}$data only, for the calculation of the hadronic vacuum polarization via a dispersion relation [84].

The contribution of SUSY particles to $\left(g_{\mu}-2\right)[85,86]$ is mainly due to neutralino-smuon and chargino-sneutrino loops [if no flavor violation is present as is the case here]. In typical models (such as mSUGRA), the contribution of chargino-sneutrino loops usually dominates. This is also true for configurations where all superpartners are almost degenerate in mass. In the latter case the contribution of $\chi_{i}^{ \pm}-\tilde{\nu}$ loops can be approximated by $[\tilde{m}$ is the mass of the heaviest particle per GeV]: $\Delta a_{\mu}^{\tilde{\chi}^{ \pm} \tilde{\nu}} \sim 10^{-5} \times\left(\tan \beta / \tilde{m}^{2}\right)$, to be compared with the contribution of $\chi_{i}^{0}-\tilde{\mu}$ loops, $\Delta a_{\mu}^{\tilde{\chi}^{0} \tilde{\mu}} \sim-10^{-6} \times\left(\tan \beta / \tilde{m}^{2}\right)$. These contributions are large for large values of $\tan \beta$ and small values of the scalar and gaugino masses. Moreover, in some other parts of the parameter space and for relatively heavy SUSY particles, the dominant SUSY effect follows the sign of the $\mu$ parameter.

We have included a routine which calculates the full one-loop contributions of charginosneutrino and neutralino-smuon loops in the MSSM, using the analytical expressions given in Ref. [86]. In this case, the full mixing in the smuon sector is of course included [this is the only place where the $A_{\mu}$ parameter plays a role in the code]. We also took into account in this evaluation the leading two-loop QED correction [87], which essentially reduce by about $\sim 7 \%$ the one-loop contribution.

In view of the above mentioned present uncertainties of the SM predictions, we refrain from providing a particular interpretation of the comparison between the experimental above value and one of the SM theoretical predictions, in terms of e.g. standard deviations from SM. Rather, the supersymmetric contributions are simply written in the SuSpect output files for any choice of input parameters and models, and can be directly compared with the (eventually updated) preferred range of the quantity $a_{\mu}^{\exp }-a_{\mu}^{\mathrm{th}, \mathrm{SM}}$. 


\subsubsection{The radiative decay $b \rightarrow s \gamma$}

Another observable where SUSY particle contributions might be large is the radiative flavor changing decay $b \rightarrow s \gamma[88,89]$. In the SM, this decay is mediated by loops containing charge $2 / 3$ quarks and $W$-bosons but in SUSY theories, additional contributions come from loops involving charginos and stops or top quarks and charged Higgs bosons [contributions from loops involving gluinos or neutralinos are very small [88] in the models considered here]. Since SM and SUSY contributions appear at the same order of perturbation theory, the measurement of the inclusive $B \rightarrow X_{s} \gamma$ decay branching ratio [41] is a very powerful tool for constraining the SUSY parameter space.

In Refs. [89,90], the next-to-leading order QCD corrections to the decay rate in the MSSM have been calculated and a FORTRAN code has been provided. It gives the most upto-date determination of $\mathrm{BR}(b \rightarrow s \gamma)$ where all known perturbative and non-perturbative effects are implemented, including all the possibly large contributions which can occur at NLO, such as terms $\propto \tan \beta$ and/or terms containing logarithms of $M_{\mathrm{EWSB}} / M_{W}$. We have interfaced this routine with our code ${ }^{7}$. Besides the fermion and gauge boson masses and the gauge couplings that we have as inputs, we will use the values of the other SM input parameters required for the calculation of the rate given in Ref. [91], except for the cut-off on the photon energy, $E_{\gamma}>(1-\delta) m_{b} / 2$ in the bremsstrahlung process $b \rightarrow s \gamma g$, which we fix to $\delta=0.9$ as in Ref. [90]. Then, again, the theoretical value of $\mathrm{BR}(b \rightarrow s \gamma)$ as calculated by the above mentioned routine is written in the output files, and can be compared by the user with the latest available data [41].

\section{Running SuSpect}

\subsection{Basic facts about SuSpect}

The program Suspect is composed of several files and routines:

i) The input files suspect2. in (standard format) or alternatively suspect2_lha.in (the SLHA format): here one can select the model to be investigated, the accuracy of the algorithm, the input data (SM fermion masses and gauge couplings). Some reasonable default values are set in the example which is provided. One would then simply select the SUSY model (pMSSM, mSUGRA, GMSB and AMSB), choose the corresponding input parameters and possibly make a few choices concerning the physical calculation (such as enforcing or not unification of the gauge couplings, changing the scale at which EWSB occurs, including or not $\mathrm{RC}$ to the masses, and choosing the routine calculating the Higgs masses). The list of possible choices is given in the next subsection.

ii) The program suspect2_call.f: this is an example of a routine which calls the main subroutine suspect2.f. This program is necessary to run SuSpect since it defines the

\footnotetext{
${ }^{7}$ We thank Paolo Gambino for providing us with his code and for his help in interfacing it with ours.
} 
primary algorithm control input parameters needed by the latter. In particular, there is an important parameter (INPUT) which controls the form of input/output: depending on its value it allows to select the input and output either in the standard (suspect2. in) or in the SLHA (suspect2_lha.in) format, or alternatively to bypass the reading of any input files, in which case all the parameters and choices are to be defined by the user in this calling routine. This last choice is particularly useful for interfacing SuSpect with other routines and/or for scans of the parameter space.

iii) The main routine suspect2.f: here all the calculation of the spectrum is performed, once the input is supplied by suspect2.in, suspect2_lha.in or suspect2_call.f. This routine is self-contained, except for the determination of the Higgs boson masses where it needs to call one additional routine for the two-loop contributions that we also supply: twoloophiggs.f (originally provided by Pietro Slavich). Note that we provide also as a separate file, needed in the compilation: bsg.f, performing the calculation of the $b \rightarrow s \gamma$ branching ratio (originally provided by Paolo Gambino).

iv) output files suspect2.out and suspect2_lha.out: these files are generated by default [they can be switched off by an appropriate value of the control parameter INPUT in suspect2_call.f] at each run of the program and gives the results for the output soft SUSY-breaking parameters [when they are calculated] and the masses and mixing angles

of the Higgs and SUSY particles. Some warnings and comments are also given when the obtained spectrum is problematic as will be discussed in the next subsection.

The routine suspect2.f consists of about 10.000 lines of code and takes about 350 Ko of memory, while the input and the calling routines have only a few hundred lines (most of them being comments). The accompanying separate routine for the calculation of the twoloop radiative correction to the Higgs masses has about 7000 lines of code. The complete executable code takes about 0.9 Mo space.

The FORTRAN files have to be compiled altogether and, running for instance on a PC using GNU FORTRAN, the (minimal options) compilation and link commands are:

g77 -c suspect2_call.f suspect2.f twoloophiggs.f bsg.f

g77 -o suspect suspect2_call.o suspect2.o twoloophiggs.o bsg.o

suspect

no other compilation option is in principle needed, though some users might find it useful to try other standard compilation options. The running time for a typical model point, for instance an mSUGRA point, is about 1 second on a PC with a $1 \mathrm{GHz}$ processor.

\subsection{The main routine and the control parameters}

In this subsection, while we will refrain from exhibiting the input and output files (some examples can be found on the web page of the program), we mention a few important features about the main routine and the control parameters that it is uses. 
is the main routine of the program, to be used as it is or to be called by any other routine (such as suspect2_call.f, as will be discussed below). It has the following four basic input control parameters:

IKNOWL: which sets the degree of control on the various parts of the algorithm. It has two possible values:

- IKNOWL=0: blind use of the program, i.e. no control on any "algorithmic" parameter. Reasonable default values are set for the control parameters and the program gives just the results from the physical input.

- IKNOWL=1: in addition warning/error messages are collected in the suspect2 . out file (this is the recommended choice in general).

INPUT: is for the physical input setting and can take the following values:

- INPUT=0: the model and option parameters ichoice(1)-(10) as well as the values of the input parameters are read off from the file suspect2.in in the original SuSpect format. The output is written in both suspect2.out and SLHA format suspect2_lha.out files.

- INPUT=1: the user defines all the relevant input choices and parameters within his calling program, i.e. any reading of input file(s) is bypassed. The required list of parameters to be defined (with consistent names etc), can be found in the commons given below. In addition, examples of input parameter setting for this option, with all the necessary input parameters (appearing in common blocks), are given for various models in the calling program suspect2_call.f. The output is written in both suspect2. out and SLHA format suspect2_lha.out files.

- INPUT=2: the model and option parameters ichoice(1)-(10) as well as the values of the physical input parameters are read off from the file suspect2_lha.in in the SLHA format. The output is written in both suspect2.out and SLHA format suspect2_lha. out files.

- INPUT=11: same as INPUT=1, but with no output file(s) written (note this option may be more convenient e.g. for scans of the MSSM parameter space).

ICHOICE: initializes the various options for the models to be considered, the degree of accuracy to be required, the features to be included, etc. There are 11 possible choices at present and the options are described in detail in the input files suspect2. in or suspect2_lha. in:

- ICHOICE(1): Choice of the model to be considered.

- ICHOICE(2): For the perturbative order (1 or 2 loop) of the RGEs.

- ICHOICE (3): To impose or not the GUT scale.

- ICHOICE (4): For the accuracy of the RGEs.

- ICHOICE(5): To impose or not the radiative EWSB.

- ICHOICE(6): To chose different input in general MSSM. 
- ICHOICE(7): For the radiative corrections to the (s)particles masses.

- ICHOICE(8): To set the value of the EWSB scale.

- ICHOICE(9): For the final accuracy in the spectrum calculation (two possibilities).

- ICHOICE(10): For the approximation in calculating the Higgs boson masses (approximate, one-loop, or two-loop).

ERRMESS: which provides a useful set of warning/error message flags, that are automatically written in the output files suspect2.out or suspect2_lha.out:

- $\operatorname{ERRMESS}(i)=0$ : Everything is fine,

- ERRMESS (1) $=-1$ : tachyonic 3rd generation sfermion from RGE,

- ERRMESS (2)=-1: tachyonic 1st/2d generation sfermion from RGE,

- ERRMESS(3)=-1: tachyonic $A$ boson (maybe temporary: see final mass),

- ERRMESS (4) $=-1$ : tachyonic 3rd generation sfermion from mixing,

- ERRMESS (5)=-1: $\mu\left(M_{\mathrm{GUT}}\right)$ guess inconsistent,

- ERRMESS (6) $=-1$ : non - convergent $\mu$ from EWSB,

- ERRMESS (7)=-1: EWSB maybe inconsistent (but RG-improved only check),

- ERRMESS (8)=-1: $V_{\text {Higgs }}$ maybe UFB or CCB (but RG-improved only check),

- ERRMESS (9)=-1: Higgs boson masses are NaN,

- ERRMESS (10)=-1: RGE problems (non-perturbative and/or Landau poles).

\subsection{Calculations with SuSpect}

\subsubsection{Comparison with other codes}

Our results for some representative points of the MSSM parameter space have been carefully cross-checked against other existing codes. Most of the earlier comparisons [94] have been performed in the context of mSUGRA type models but, more recently, comparisons in other models have been performed. We obtain, in general, a very good agreement, at the percent level and often better [95], with the codes SoftSusy and SPheno ${ }^{8}$. The most sophisticated parameter to obtain in this context is the lightest Higgs boson mass, since it incorporates all possible ingredients: the RGE's for the evaluation of $M_{H_{u}}$ and $M_{H_{d}}$, the effective potential and the EWSB for the determination of $M_{A}$ and the tadpoles, the radiative corrections to the Higgs sector which involve also the two-loop corrections, etc. Recently, a detailed comparison of SuSpect with SoftSusy and SPheno (and also FeynHiggs) for the Higgs boson masses in many scenarios (mSUGRA, GMSB, AMSB, general MSSM) was performed in Ref. [34]. The value that we obtain for $M_{h}$, for instance, is in a very good agreement, the difference being less than half a GeV, while the agreement for $M_{A}$ and $\mu$ is at the level of $1 \%$; see Ref. [34] for details. The agreement with the program ISASUGRA is not as good, in particular for what concerns the Higgs sector, since different approximations have been used in the two cases.

\footnotetext{
${ }^{8}$ We thank Ben Allanach, Sabine Kraml, Werner Porod and Pietro Slavich for their gracious help in performing these detailed comparisons of the programs.
} 


\subsubsection{Interface with other programs}

In the way it is written, SuSpect can be easily interfaced with other programs or MonteCarlo event generators ${ }^{9}$. Private or official versions of some programs exist in which an interface with SuSpect has been made; we give a short list of them ${ }^{10}$ :

- micrOMEGAs [96]: for the automatic (analytical and then numerical) calculation of the cosmological relic density of the lightest neutralinos, including all possible channels.

- DARKSUSY [97]: also for the calculation of the relic density of the lightest neutralinos [including co-annihilation effects] and their direct and indirect detection rates as well as other astrophysical features. [The program has its own calculation of the SUSY spectrum, but it is rather approximate $]^{11}$.

- HDECAY [76]: for the calculation of the decay branching ratios and total decay widths of the SM and MSSM Higgs bosons [in fact some routines, in particular those for the QCD running and for the interface with the routines calculating the Higgs boson masses, are borrowed from there].

- SDECAY [100]: for the calculation of the decay widths and branching ratios of SUSY particles including higher order [three-body decays for gauginos and stops, four-body decays for the lightest stop and QCD corrections to the two-body decays of squarks and gluinos], which will appear soon.

- SUSYGEN [101]: a Monte-Carlo event generator for Higgs and SUSY particle production in the MSSM [mainly in $e^{+} e^{-}$collisions but some processes in $e p$ and $p p$ collisions are implemented]. The program is also interfaced with HDECAY.

- SFITTER [102] which determines the weak scale MSSM parameters from measurements performed at hadron and $e^{+} e^{-}$colliders from a combined fit/grid taking into account the correlations.

As discussed already, we also interfaced SuSpect with the code bsg.f which calculate the rate of the $b \rightarrow s \gamma$ decay at next-to-leading order [90]. An interface with the MonteCarlo event generators such as PYTHIA [103] and HERWIG [104] can be made throgh the SLHA format.

\footnotetext{
${ }^{9}$ To make this interfacing easier, in addition to including the input and output files in the now standard SLHA format, we also have provided a set of obvious commons for the input and output parameters needed or calculated by SuSpect and named all commons, subroutines and functions used with a prefix SU_, in order not to be in conflict with those used by other programs.

${ }^{10}$ Note that we have also interfaced the program with a private code written by Manuel Drees calculating the cosmological relic density of the lightest neutralinos for the complete analysis of the mSUGRA parameter space performed in Ref. [92].

${ }^{11}$ Note that a private Suspect/DARKSUSY interface has been already used for prediction studies of indirect LSP detection $[98,99]$.
} 


\subsubsection{Web information and maintenance}

A web page devoted to the SuSpect program can be found at the http address:

$$
\text { http://www.lpta.univ-montp2.fr/ } \text { kneur/Suspect }
$$

It contains all the information that one needs on the program:

- Short explanations of the code and how to run it.

- The complete "users manual" can be obtained in post-script or PDF form.

- A list of important changes/corrected bugs in the code.

- A mailing list to which one can subscribe to be automatically advised about future SuSpect updates or eventual corrections.

One can also download directly the various files of the program:

- suspect2.in, suspect2_lha.in: the input files in standard or SLHA format.

- suspect2_call.f: the calling program sample.

- suspect2.f: the main routine of the program.

- twoloophiggs.f: the additional Higgs routine needed.

- SuSpect2.tar.gz: all needed routines in standard compressed format for the latest version.

- SuSpect2_0ld.uu: the routines for the previous versions of the program.

Note that the possibility to use directly the program [as well as the other RGE codes] interactively on the web has been set by Sabine Kraml [95].

\section{Conclusion}

We have presented the version 2.3 of the ForTRAn code SuSpect which calculates the Supersymmetric and Higgs particle spectrum in the MSSM. The calculation can be performed in constrained models with universal boundary conditions at high scales such as the gravity (mSUGRA), anomaly (AMSB) or gauge (GMSB) mediated breaking models, but also in the non-universal or unconstrained MSSM case, with up to 22 free input parameters which can be set either at the electroweak symmetry breaking scale or obtained from boundary conditions on some common parameters at a high-energy scale.

A particular care has been taken to treat all the mandatory features which are needed to describe accurately these various scenarii: the renormalization group evolution of parameters between low and high energy scales, the consistent implementation of radiative electroweak symmetry breaking and the calculation of the physical masses of the Higgs bosons and supersymmetric particles taking into account all dominant radiative corrections. The program provides several options [for accuracy, scale choice, etc...] to deal with these aspects.

The program can check the fulfillment of theoretical constraints, such as the absence of tachyonic particles and improper lightest SUSY particle, the absence of non desired charge and color breaking as well as unbounded from below minima and a large fine-tuning in the 
electroweak symmetry breaking conditions. A verification that the obtained spectrum is in agreement with high precision measurements such as the $\rho$ parameter, the muon $g-2$ and the radiative $b \rightarrow s \gamma$ decay, can also be performed.

The program has a high degree of flexibility in the choice of the model and/or the input parameters and an adequate level of approximation at different stages. It is rather precise and quite reliable [since it has been compared with several other similar existing codes], relatively fast to allow for rapid comprehensive scans of the parameter space and simple enough to be linked with other programs dealing with MSSM particle properties or with Monte-Carlo event generators. We have also provided a simpler way to run the code interactively on the web in the constrained models.

Several upgrades, which include the possibility to analyze additional theoretical models and to further extend the already existing interfaces with programs for (s)particle decay branching ratios and Dark Matter calculations and with some Monte-Carlo event generators to simulate the production properties, are planned.

\section{Acknowledgments:}

In its latest present version, the code SuSpect benefited largely from the invaluable help and many cross-checks of Pietro Slavich, in particular, in implementing a consistent interface with his routine for the $\overline{\mathrm{DR}}$ calculation of the Higgs masses including the leading two-loop contributions. In former stages the code has been developed in the framework of the French working group (GDR-SUSY), organized by the Centre National de la Recherche Scientifique (CNRS), and has been checked and "debugged" during the last few years with the help of several members of the "MSSM" and "Tools" working groups of the GDR to whom we are indebted. We thank in particular, Genevieve Bélanger, Fawzi Boudjema, Marie-Bernadette Causse, Jean-Baptiste de Vivie, Laurent Duflot, Nabil Ghodbane, JeanFrancois Grivaz, Cyril Hugonie, Stavros Katsanevas, Vincent Lafage, Rémi Lafaye, Imad Laktineh, Christophe Le Mouël, Yann Mambrini, Steve Muanza, Margarete Mühlleitner, Emmanuel Nezri, Jean Orloff, Emmanuelle Perez, Sylvie Rosier-Lees, Roberto Ruiz de Austri, Dirk Zerwas. We have as well similarly benefited from the working group "SUSY Dark Matter" of the GDR Phénomènes Cosmiques de Hautes Energies. We thank all members of this group, in particular Julien Guy, Agnieszka Jacholkowska, Julien Lavalle, Eric Nuss and Mariusz Sapinski for various cross-checks. We have also benefited from several discussions, comments and help from: Ben Allanach, Pierre Binétruy, Andreas Birkedal, Francesca Borzumati, Aseshkrishna Datta, Manuel Drees, Paolo Gambino, Naveen Gaur, Sven Heinemeyer, Jan Kalinowski, Yeong Gyun Kim, Sabine Kraml, Filip Moortgat, Stefano Moretti, Takeshi Nihei, Werner Porod, François Richard, Peter Richardson, Leszek Roszkowski, Aurore Savoy-Navarro, Peter Skands, Michael Spira and Charling Tao. We thank them all. Finally, thanks to our system-manager in Montpellier, Dominique Caron, for technical help. 


\section{References}

[1] For an introduction, see: "Supersymmetry and Supergravity" by J. Wess and J. Bagger, Princeton Series in Physics.

[2] For reviews on the MSSM, see: H.P. Nilles, Phys. Rep. 110 (1984) 1; R. Barbieri, Riv. Nuov. Cim. 11 (1988) 1; R. Arnowitt and Pran Nath, Report CTP-TAMU52-93; M. Drees and S.P. Martin, hep-ph/9504324; J. Bagger, Lectures at TASI-95, hep-ph/9604232 S.P. Martin, hep-ph/9709356 S. Dawson, hep-ph/9712464.

[3] H. E. Haber and G. Kane, Phys. Rep. 117 (1985) 75; J.F. Gunion and H.E. Haber, Nucl. Phys. B272 (1986) 1 and Nucl. Phys. B278 (1986) 449 ; (E) hep-ph/9301205.

[4] A. Djouadi and S. Rosiers-Lees et al, Summary Report of the MSSM Working Group for the "GDR-Supersymétrie", hep-ph/9901246.

[5] For a review on the Higgs sector of the MSSM, see J.F. Gunion, H.E. Haber, G.L. Kane and S. Dawson, "The Higgs Hunter's Guide", Addison-Wesley, Reading 1990.

[6] M. Carena et al., Report of the Higgs WG hep-ph/0010338 and S. Abel et al, Report of the MSSM WG hep-ph/0003154, for "RUN II at the Tevatron"; CMS Coll., Technical Proposal, report CERN/LHCC/94-38 (1994); ATLAS Coll., Technical Design Report, CERN/LHCC/99-15 (1999); Proceedings of the Les Houches Workshops: A. Djouadi et al., hep-ph/0002258 (1999) and D. Cavalli et al., hep-ph/0203056 (2001); E. Accomando, Phys. Rept. 299 (1998) 1; American Linear Collider Working Group, hep-ex/0106057; ACFA Linear Collider Working group hep-ph/0109166 TESLA Technical Design Report, Part III, hep-ph/0106315.

[7] S. Dimopoulos and D. Sutter, Nucl. Phys. B452 (1995) 496; see also the discussions given by H.E. Haber, hep-ph/9709450 and G.L. Kane, hep-ph/0008190

[8] A.H. Chamseddine, R. Arnowitt and P. Nath, Phys. Rev. Lett. 49 (1982) 970; R. Barbieri, S. Ferrara and C.A Savoy, Phys. Lett. B119 (1982) 343; L. Hall, J. Lykken and S. Weinberg, Phys. Rev. D27 (1983) 2359.

[9] L. Randall and R. Sundrum, Nucl. Phys. B557 (1999) 79; G. Giudice, M. Luty, H. Murayama and R. Rattazzi, JHEP 9812 (1998) 027.

[10] J.A. Bagger, T. Moroi and E. Poppitz, JHEP 0004 (2000) 009.

[11] For a recent phenomenological review of AMSB models, see for instance: K. Huitu, J. Laamanen and P. N. Pandita, Phys. Rev. D65 (2002) 115003.

[12] For a general review: G.F. Giudice and R. Rattazzi, Phys. Rept. 322 (1999) 419. 
[13] For phenomenological reviews of GMSB models, see for instance: S. Ambrosanio, G.D. Kribs and S.P. Martin, Phys. Rev. D56 (1997) 1761; C.H. Chen and J.F. Gunion, Phys. Rev. D58 (1998) 075005.

[14] H. Baer, F.E. Paige S.D. Protopopescu and X. Tata, "ISAJET", hep-ph/0001086.

[15] B.C. Allanach, "SOFTSUSY", Comput. Phys. Commun. (2002) 143.

[16] W. Porod, "SPHENO”, Comput. Phys. Commun. 153 (2003) 275.

[17] A preliminary version of the program exists since 1998 and was described in A. Djouadi, J.L. Kneur and G. Moultaka in Ref. [4].

[18] K. Inoue, A. Kakuto, H. Komatsu and S. Takeshita, Prog. Theor. Phys. 68, 927 (1982); Erratum: ibid. 70, 330 (1983); ibid. 71, 413 (1984); M. Machacek and M.T. Vaughn, Nucl. Phys. B222 (1983) 83; ibid. B236 (1984) 221; ibid B249 (1985) 70; I. Jack, Phys. Lett. B147 (1984) 405; W. de Boer, R. Ehret and D.I. Kazakov, Z. Phys. C67 (1994) 647; Y. Yamada, Phys. Rev. D50 (1994) 3537; I. Jack and D.R.T. Jones, Phys. Lett. B333 (1994) 372.

[19] D.J. Castaño, E.J. Piard and P. Ramond, Phys. Rev. D49 (1994) 4882; V. Barger, M.S. Berger and P. Ohmann, Phys. Rev. D47 (1993) 1093.

[20] I. Jack, D. Jones, S. Martin, M. Vaughn and Y. Yamada, Phys. Rev. D50 (1994) 5481; S. Martin and M. Vaughn, Phys. Lett. B318, 331 (1993) and Phys. Rev. D50 (1994) 47.

[21] K. Inoue et al. in Ref. [18], L. Ibañez and G.G. Ross Phys. Lett. B110 (1982) 227; L. Alvarez-Gaumé, J. Polchinski and M.B. Wise, Nucl. Phys. B221 (1983) 495; J. Ellis, J. Hagelin, D. Nanopoulos and K. Tamkavis, Phys. Lett. B125 (1983) 275, L.E. Ibanez and C. Lopez, Nucl. Phys. B233 (1984) 511; L.E. Ibanez, C. Lopez and C. Munoz, Nucl. Phys. B250 (1985) 218.

[22] R. Arnowitt and P. Nath, Phys. Rev. D46, 3981 (1992); V. Barger, M.S. Berger and P. Ohmann, Phys. Rev. D49, 4908 (1994); P.H. Chankowski, S. Pokorski and J. Rosiek, Nucl. Phys. B423 (1994) 437.

[23] G. Gamberini, G. Ridolfi and F. Zwirner, Nucl. Phys. B331, 331 (1990); B. de Carlos and J.A. Casas, Phys. Lett. B309, 320 (1993).

[24] A.B. Lahanas and V.C. Spanos, Eur. Phys. J. C23 (2002) 185.

[25] D.M. Pierce, J.A. Bagger, K. Matchev and R.J. Zhang, Nucl. Phys. B491 (1997) 3. 
[26] For some earlier work on SUSY radiative corrections is for instance: J.A. Grifols and J. Sola, Phys. Lett. B137 (1984) 257 and Nucl. Phys. B253 (1985) 47; P. Chankowski et al., Nucl. Phys. B417 (1994) 101; D. Pierce and A. Papadopoulos, Nucl. Phys. B430 (1994) 278 and Phys. Rev. D50 (1994) 565; A. Donini, Nucl. Phys. B467 (1996) 3; N. Krasnikov, Phys. Lett. B345 (1995) 25; A.B. Lahanas, K. Tamkavis and N.D. Tracas, Phys. Lett. B324 (1994) 387; M. Drees, M.M. Nojiri, D.P. Roy and Y. Yamada, Phys. Rev. D56 (1997) 276.

[27] For radiative corrections in the Higgs sector, see for instance: Y. Okada, M. Yamaguchi and T. Yanagida, Prog. Theor. Phys. 85 (1991) 1; H. Haber and R. Hempfling, Phys. Rev. Lett. 66 (1991) 1815; J. Ellis, G. Ridolfi and F. Zwirner, Phys. Lett. 257B (1991) 83; R. Barbieri, F. Caravaglios and M. Frigeni, Phys. Lett. 258 B (1991) 167; M. Drees and M.M. Nojiri, Phys. Rev. D45 (1992) 2482; R. Hempfling and A. Hoang, Phys. Lett. B331 (1994) 99; M. Carena, J. Espinosa, M. Quiros and C. Wagner, Phys. Lett. B335 (1995) 209; M. Carena, M. Quiros and C.E.M. Wagner, Nucl. Phys. B461 (1996) 407; H. Haber, R. Hempfling and A. Hoang, Z. Phys. C75 (1997) 539; S. Heinemeyer, W. Hollik and G. Weiglein, Phys. Rev. D58 (1998) 091701; J.R. Espinosa and R.J. Zhang, JHEP0003 (2000) 026 and Nucl. Phys. B586 (2000)3; M. Carena et al., Nucl. Phys. B580 (2000) 29.

[28] S. Heinemeyer, W. Hollik and G. Weiglein, Comput. Phys. Commun. 124 (2000) 76.

[29] S. Heinemeyer, W. Hollik and G. Weiglein, hep-ph/0002213.

[30] G. Degrassi, P. Slavich and F. Zwirner, Nucl. Phys. B611 (2001) 403.

[31] A. Brignole, G. Degrassi, P. Slavich and F. Zwirner, Nucl. Phys. B631 (2002) 195.

[32] A. Brignole, G. Degrassi, P. Slavich and F. Zwirner, Nucl. Phys. B643 (2002) 79.

[33] A. Dedes, G. Degrassi and P. Slavich, Nucl. Phys. B672 (2003) 144.

[34] B.C. Allanach, A. Djouadi, J.-L. Kneur, W. Porod and P. Slavich, JHEP 0409 (2004) 044.

[35] P. Skands et al., JHEP 0407 (2004) 036.

[36] P. Fayet, Phys. Lett. 69B (1977) 489; G. Farrar and P. Fayet, Phys. Lett. $76 B$ (1978) 575; N. Sakai, Z. Phys. C11 (1981) 153; S. Dimopoulos and H. Georgi, Nucl. Phys. B193 (1981) 150; K. Inoue, A. Komatsu and S. Takeshita, Prog. Theor. Phys 68 (1982) 927; E. Witten, Nucl. Phys. B231 (1984) 419.

[37] P. Fayet and J. Iliopoulos, Phys. Lett. 51B (1974) 461; L. O’Raifeartaigh, Nucl. Phys. B96 (1975) 331. 
[38] L. Girardello and M.T. Grisaru, Nucl. Phys. B194 (1982) 65.

[39] For a review, see: A. Masiero and L. Silvestrini, hep-ph/9711401.

[40] J.M. Frere, D.R.T. Jones and S. Raby, Nucl. Phys. B222 (1983) 11; L. Alvarez-Gaumé, J. Polchinski and M.B. Wise, Nucl. Phys. B221 (1983) 495; J.P. Derendiger and C.A. Savoy, Nucl. Phys. B237 (1984) 307; C. Kounas, A.B. Lahanas and M. Quiros, Nucl. Phys. B236 (1984) 438; M. Claudson, L.J. Hall and I. Hinchliffe, Nucl. Phys. B236 (1983) 501.

[41] Particle Data Group, S. Eidelman et al, Phys. Lett. B592, 1 (2004).

[42] J. Ellis, S. Kelley and D.V. Nanopoulos, Phys. Lett. B260 (1991) 131; U. Amaldi, W. de Boer and H. Fürstenau, Phys. Lett. B260 (1991) 447; P. Langacker and M. Luo, Phys. Rev.D 44 (1991) 817; C. Giunti, C.W. Kim and U.W. Lee, Mod. Phys. Lett. A6 (1991) 1745.

[43] T. Gherghetta, G.F. Giudice, J.D. Wells, Nucl. Phys. B559 (1999) 27.

[44] See e.g., D.E. Kaplan and G.D. Kribs, JHEP 09 (2000) 048.

[45] See e.g., M. Carena, K. Huitu and T. Kobayashi, Nucl. Phys. B592 (2001) 164.

[46] M. Dine and A. E. Nelson, Phys. Rev. D48 (1993) 1277; M. Dine, A. E. Nelson and Y. Shirman, Phys. Rev. D51 (1995) 1362; M. Dine, A. E. Nelson, Y. Nir and Y. Shirman, Phys. Rev. D53 (1996) 2658.

[47] N. Arkani-Hamed, J. March-Russell and H. Murayama, Nucl. Phys. B509 (1998) 3; H. Murayama, Phys. Rev. Lett. 79 (1997) 18; K.I. Izawa, Y. Nomura, K. Tobe and T. Yanagida, Phys. Rev. D56 (1997) 2886; M.A. Luty, Phys. Lett. B414 (1997) 71.

[48] R. Barbieri, G. Dvali and A. Strumia, Phys. Lett. B333 (1994) 79; E. Witten, hep-ph/0201018

[49] K. Agashe and M. Graesser, Nucl. Phys. B507 (1997) 34.

[50] S.K. Soni and H.A. Weldon, Phys. Lett. 126B (1983) 215.

[51] L.E. Ibáñez and D. Lust, Nucl. Phys. B382 (1992) 305.

[52] See e.g., P. Binetruy, M.K. Gaillard and B.D. Nelson, Nucl. Phys. B604 (2001) 32.

[53] For phenomenological discussions and references on the original models, see for instance: G. Anderson et al., hep-ph/9609457 and H. Baer et al., JHEP 0205 (2002) 061. 
[54] See e.g., G. Cohen, D.B. Kaplan and A.E. Nelson, Phys. Lett. B388 (1996) 588; S. Dimopoulos and G.F. Giudice, Phys. Lett. B357 (1995) 573; A. Pomarol and D. Tommasini, Nucl. Phys. B466(1996) 3; P. Binetruy and E. Dudas, Phys. Lett. B389 (1996) 503; B. de Carlos, J. Casas, F. Quevedo and E. Roulet, Phys. Lett. B318 (1993) 447; J. Bagger, J.L. Feng and N. Polonsky, Nucl. Phys. B563 (1999) 3; J. Bagger, J.L. Feng, N. Polonsky and R.J. Zhang, Phys. Lett. B473 (2000) 264; V.D. Barger, C. Kao and R.J. Zhang, Phys. Lett. B483 (2000) 184.

[55] M. Drees, Phys. Lett. B181 (1986) 279.

[56] C. Kolda and S. Martin, Phys. Rev. D53 (1996) 3871.

[57] D. Matalliotakis and H.P. Nilles, Nucl. Phys. B435 (1995) 115; M. Olechowski and S. Pokorski, Phys. Lett. B344 (1995) 201; N. Polonsky and A. Pomarol, Phys. Rev. D51 (1995) 6352; V. Berenzenski et al., Astropart. Phys. 5 (1996) 1; R. Arnowitt and P. Nath, Phys. Rev. D56 (1997) 2820 and 2833.

[58] Example of analyses where Higgs and sfermion masses are disconnected are: G. Belanger et al., Nucl. Phys. B581 (2000) 3; A. Datta et al., Phys. Rev. D 65 (2002) 015007; A. Djouadi et al., Phys. Lett. B376 (1996) 220 (1996) and Eur. Phys. J. C1 (1998) 149.

[59] V.D. Barger, F. Halzen, D.Hooper and Chung Kao, Phys. Rev. D65 (2002) 075022; V. Bertin, E. Nezri and J. Orloff JHEP 0302 (2003) 046; J. Ellis, T. Falk, K.A. Olive and Y. Santoso, Nucl.Phys. B652 (2003) 259; A. Birkedal-Hansen and B. Nelson, Phys.Rev. D67 (2003) 095006.

[60] See e.g., M. Carena, M. Quiros and C.E.M. Wagner, Nucl. Phys. B524 (1998) 3.

[61] A. Djouadi, J.L. Kneur, G. Moultaka, Phys. Rev. Lett. 80 (1998) 1830 and Nucl. Phys. B569 (2000) 53; A. Djouadi, Phys. Lett. B435 (1998) 101; C. Boehm et al., Phys. Rev. D61 (2000) 095006 and Phys. Rev. D62 (2000) 035012.

[62] For the pure QCD corrections to the quark masses, we follow the approach of A. Djouadi, M. Spira and P.M. Zerwas, Z. Phys. C70 (1996) 427, which has been also used in the program HDECAY, except that we work in the $\overline{\mathrm{DR}}$ scheme.

[63] N. Gray, D.J. Broadhurst, W. Grafe and K. Schilcher, Z. Phys. C48 (1990) 673.

[64] S.G. Gorishny, A.L. Kataev, S.A. Larin and L.R. Surguladze, Mod. Phys. Lett. A5 (1990) 2703; Phys. Rev. D43 (1991) 1633.

[65] L.V. Avdeev and M.Yu. Kalmykov, Nucl. Phys. B502 (1997) 419; H. Baer, J. Ferrandis, K. Melnikov and X. Tata, Phys. Rev. D66 (2002) 074007. 
[66] M. Carena, D. Garcia, U. Nierste and C. E. Wagner, Nucl. Phys. B577 (2000) 88 hep-ph/9912516;

[67] T. Banks, Nucl. Phys. B303 (1988) 172; L. J. Hall, R. Rattazzi and U. Sarid, Phys. Rev. D50 (1994) 7048; R. Hempfling, Phys. Rev. D49 (1994) 6168; M. Carena, M. Olechowski, S. Pokorski and C. E. Wagner, Nucl. Phys. B426 (1994) 269; T. Blazek, S. Pokorski and S. Raby, Phys. Rev. D52 (1995) 4151; H. Eberl, K. Hidaka, S. Kraml, W. Majerotto and Y. Yamada, Phys. Rev. D62 (2000) 055006.

[68] The Tevatron Electroweak Working Group [D0 Collaboration], hep-ex/0404010.

[69] J. Hisano, H. Murayama and T. Yanagida, Nucl. Phys. B402 (1993) 46, hep-ph/9207279; Y. Yamada, Z. Phys. C60 (1993) 83; G.G. Ross and R.G. Roberts, Nucl. Phys. B377 (1992) 571.

[70] Stephen P. Martin, Phys. Rev. D66 (2002) 096001.

[71] J.A. Casas, A. Lleyda and C. Muñoz, Nucl. Phys. B471 (1996) 3.

[72] C. Le Mouël, Phys. Rev. D 64 (2001) 075009 and Nucl. Phys. B 607 (2001) 38.

[73] A. Kusenko, P.Langacker and G. Segre, Phys. Rev. D54 (1996) 5824.

[74] J. Ellis and S. Rudaz, Phys. Lett. B128 (1983) 248; M. Drees and K. Hikasa, Phys. Lett. B252 (1990) 127.

[75] M.M. El Kheishen, A.A. Shafik and A.A. Aboshousha, Phys. Rev. D45, 4345 (1992); M. Guchait, Z. Phys. C57, 157 (1993), Erratum: ibid. C61, 178 (1994).

[76] A. Djouadi, J. Kalinowski and M. Spira, Comput. Phys. Commun. 108 (1998) 56.

[77] R. Arnowitt and P. Nath, Phys. Lett. B289 (1992) 368; S. Kelley et al., Nucl. Phys. B398 (1993); Olechowski and S. Pokorski, Nucl. Phys. B404 (1993) 590; B. de Carlos and J.A. Casas, Nucl. Phys. B309 (1993) 320; G.G. Ross and R.G. Roberts in [69].

[78] R. Barbieri and G. Giudice, Nucl. Phys. B306 (1988) 63; for a more recent discussion see, K. Agashe and M. Graesser in Ref. [49].

[79] M. Veltman, Nucl. Phys. B123 (1977) 89; A. Djouadi and C. Verzegnassi, Phys. Lett. B195 265 (1987); A. Djouadi, Nuov. Cim. A100, 357 (1988); B.A. Kniehl, Nucl. Phys B347 (1990) 86; A. Djouadi and P. Gambino, Phys. Rev. D49 (1994) 3499 and Phys. Rev. D51 (1995) 218; K. Chetyrkin, J. Kühn and M. Steinhauser, Phys. Rev. Lett. 75, 3394 (1995); L. Avdeev et al., Phys. Lett. B336, 560 (1994); G. Degrassi, P. Gambino and A. Vicini, Phys. Lett. B383 (1996) 219; A. Freitas et al., Nucl. Phys. B632 (2002) 189; M. Awramik and M. Czakon, Phys. Rev. Lett. 89 (2002) 241801; M. Awramik, M. Czakon, A. Onishchenko and O. Veretin, Phys. Rev. D68 (2003) 053004. 
[80] R. Barbieri and L. Maiani, Nucl. Phys. B224, 32 (1983); C.S. Lim, T. Inami and N. Sakai, Phys. Rev. D29, 1488 (1984); E. Eliasson, Phys. Lett. 147B, 65 (1984); M. Drees and K. Hagiwara, Phys. Rev. D42 (1990) 1709; M. Drees, K. Hagiwara and A. Yamada, Phys. Rev. D45, 1725, (1992); P. Chankowski, A. Dabelstein, W. Hollik, W. Mösle, S. Pokorski and J. Rosiek, Nucl. Phys. B417 (1994) 101; D. Garcia and J. Solà, Mod. Phys. Lett. A 9 (1994) 211.

[81] A. Djouadi, P. Gambino, S. Heinemeyer, W. Hollik, C. Jünger and G. Weiglein, Phys. Rev. Lett. 78 (1997) 3626 and Phys. Rev. D57 (1998) 4179.

[82] The LEP Collaborations (ALEPH, L3, DELPHI and OPAL), hep-ex/0103048.

[83] Muon $g-2$ Collab., G.W. Bennett et al., Phys. Rev. Lett. 89, 101804 (2002), Erratumibid. 89, 129903 (2002), hep-ex/0208001, and Phys. Rev. Lett. 92, 161802 (2004), hep-ex/0401008

[84] For a recent discussion of theoretical and experimental developments including the calculation of M. Knecht and A. Nyffeler, Phys. Rev. D65 (2002) 073034, see: M. Davier, S. Eidelman, A. Hocker and Z. Zhang, Eur. Phys. J. C27 (2003) 497.

[85] J. Ellis, J. Hagelin and D.V. Nanopoulos, Phys. Lett. 116B, 283 (1982); J.A. Grifols and A. Mendez, Phys. Rev. D26, 1809 (1982); R. Barbieri and L. Maiani, Phys. Lett. 117B, 203 (1982); D.A. Kosower, L.M. Krauss and N. Sakai, Phys. Lett. 133B, 305 (1983); U. Chattopadhyay and P. Nath, Phys. Rev. D53 (1996) 1648; T. Moroi, Phys. Rev. D53 (1996) 6565, and Erratum: ibid D56, 4424 (1997); M. Carena, G. Giudice and C.E. Wagner, Phys. Lett. B390 (1997) 234.

[86] S.P. Martin and J.D. Wells Phys. Rev. D64 (2001) 035003.

[87] G. Degrassi and G.F. Giudice, Phys. Rev. D58 (1998) 053007.

[88] S. Bertolini, F. Borzumati, A. Masiero and G. Ridolfi, Nucl. Phys. B353, 591 (1991); R. Barbieri and G. Giudice, Phys. Lett. B309, 86 (1993); F. Borzumati, M. Olechowski and S. Pokorski, Phys. Lett. B349 (1995) 311.

[89] M. Ciuchini, G. Degrassi, P. Gambino and G.F. Giudice, Nucl. Phys. B534 (1998) 3; C. Bobeth, M. Misiak and J, Urban, Nucl. Phys. B567 (2000) 153; F. Borzumati, C. Greub, T. Hurth and D. Wyler, Phys. Rev. D62 (2000) 075005.

[90] G. Degrassi, P. Gambino and G.F. Giudice, JHEP 0012 (2000) 009.

[91] M. Ciuchini, G. Degrassi, P. Gambino and G.F. Giudice, Nucl. Phys. B527 (1998) 21.

[92] A. Djouadi, M. Drees and J.L. Kneur, JHEP 0108 (2001) 055. 
[93] B.C. Allanach et al., "The Snowmass points and slopes: Benchmarks for SUSY searches", Eur. Phys. J. C25 (2002) 113 hep-ph/0202233; N. Ghodbane and H.U. Martyn, hep-ph/0201233.

[94] B. Allanach, S. Kraml and W. Porod, "Comparison of SUSY mass spectrum calculations", hep-ph/0207314 JHEP 0303 (2003) 016 hep-ph/0302102.

[95] See the web site: http://cern.ch/kraml/comparison/

[96] G. Bélanger, F. Boudjema, A. Pukhov and A. Semenov, "MicrOMEGAs: A Program for calculating the relic density in the MSSM", Comput. Phys. Commun. 149 (2002) 103.

[97] P. Gondolo, J. Edsjo, L. Bergstrom, P. Ullio and E. Baltz, DarkSUSY: A Numerical package for dark matter calculations in the MSSM", astro-ph/0012234.

[98] V. Bertin, E. Nezri, J. Orloff, Eur. Phys. J. C26 (2002) 111.

[99] A. Falvard, E. Giraud, A. Jacholkowska, J. Lavalle, E. Nuss, F. Piron, M. Sapinski, P. Salati, R. Taillet, K. Jedamzik and G. Moultaka, Astropart. Phys. 20 (2004) 467; see also E. Giraud et al. astro-ph/0209230, in Astronomy, Cosmology and Fundamental Physics proceedings of ESO-CERN-ESA Symposium eds. P. A. Shaver, L. Di Lella, and A. Gimenez.

[100] A. Djouadi, Y. Mambrini and M. Muhlleitner, "SDECAY", hep-ph/0311167.

[101] S. Katsanevas and P. Morawitz, "SUSYGEN", Comput. Phys. Commun. 112 (1998) 227; E. Perez, in "Hamburg 1998/1999, Monte Carlo generators for HERA physics", p. 635-651.

[102] R. Lafaye, T. Plehn and D. Zerwas, SFITTER, hep-ph/0404282.

[103] T. Sjostrand, L. Lonnblad and S. Mrenna, "PYTHIA", hep-ph/0108264.

[104] G. Corcella et al., "HERWIG 6", JHEP 0101 (2001) 010. 